\title{
Multi-dimensional Limiting Strategy for Higher-order CFD Methods - Progress and Issue (Invited)
}

\author{
Chongam Kim* \\ Department of Aerospace Engineering, Seoul National University, Seoul, 151-744, Korea \\ Jin Seok Park ${ }^{\dagger}$ \\ Department of Aeronautics, Imperial College London, London, SW7 2AZ, UK
}

\begin{abstract}
The present paper deals with the progress of multi-dimensional limiting process (MLP) and discuss the issues for further improvements. MLP, which has been originally developed in finite volume method (FVM), provides an accurate, robust and efficient oscillationcontrol mechanism in multiple dimensions for linear reconstruction. This limiting philosophy can be hierarchically extended into higher-order $P n$ approximation or reconstruction. The resulting algorithm, called the hierarchical MLP, facilitates the capturing of detailed flow structures while maintaining the formal order-of-accuracy in smooth region and providing accurate non-oscillatory solutions across discontinuous region. This algorithm has been developed within the modal DG framework, but it also can be formulated into a nodal framework, most notably the CPR framework. Troubled-cells are detected by applying the MLP concept, and the final accuracy is determined by the projection procedure and the hierarchical MLP limiting step. Through extensive numerical analyses and computations ranging from scalar conservation laws to fluid systems, it is demonstrated that the proposed limiting approach yields the outstanding performances in capturing compressible inviscid and viscous flow features. Further issues are also mentioned to improve and extend the current approach for higher-order simulations of high-Reynolds number compressible flows.
\end{abstract}

\section{Introduction}

Up to now, second-order accurate computational fluid dynamics (CFD) methods with some discontinuitycapturing strategy are widely used to analyze compressible viscous flows. Theoretically, these approaches guarantee the minimal accuracy to recover essential physics of high-Reynolds number compressible flows. Indeed, they have witnessed remarkable successes in many classes of engineering and scientific applications. At the same time, however, they also unveil some limitations, particularly in capturing unsteady vortexdominated flow structures due to excessive numerical diffusion. From this perspective, higher-order methods are convincing alternatives in the sense that they can provide the detailed flow structures by upgrading the accuracy of spatial and temporal discretization with reasonable computational resources. ${ }^{1-4}$

During the past few decades, various higher-order discretization methods have been developed. In order to handle complex geometry, these methods usually combine the merits of both finite volume methods (FVM) and finite element methods (FEM), which makes it possible to develop higher-order approximation/reconstruction for each cell with a minimal stencil. Discontinuous Galerkin (DG) method is one of the widely-used and well-developed higher-order methods in hyperbolic conservation laws. Strong mathematical background and numerical analysis support it though overall formulations are relatively complex and expensive compared to FVM. Recently, Huynh proposed the flux reconstruction (FR) procedure, ${ }^{5,6}$ which provides an unifying framework of many higher-order methods. In addition, Wang introduced a lifting collocation operator $^{7}$ to deal with multi-dimensional problems on simplex and mixed grids. The two approaches were combined and renamed as correction procedure via reconstruction (CPR), which possesses many merits of

*Professor, Department of Aerospace Engineering, Institute of Advanced Aerospace Technology, Seoul National University, AIAA Associate Fellow, Corresponding author: chongam@snu.ac.kr.

${ }^{\dagger}$ Post-doctoral Researcher, Department of Aeronautics, Imperial College London, AIAA Member. 
higher-order methods in terms of accuracy and stability, while it is more simple and efficient. Recent studies also show some encouraging results in capturing compressible flow features. ${ }^{4}$ Furthermore, the energy stability of FR was rigorously studied by Vincent et al., and as a result, energy stable flux reconstruction (ESFR) was developed. ${ }^{8,9}$

At the same time, a few obstacles have surfaced in extending higher-order CFD methods, including CPR, into high speed unsteady flows. One of them is to design a robust and efficient oscillation-control algorithm to suppress unwanted oscillations around discontinuities without compromising the higher-order nature in smooth region. The main reason for such oscillations is the lack of providing a proper diffusion mechanism. Regarding this issue, two options are currently available. One is to add a properly tuned artificial viscosity term around shock region. ${ }^{10,11}$ The other resorts to some limiting algorithms which are mostly borrowed from finite volume methods.

Cockburn and Shu developed the first successful TVB-based limiter for the DG method, ${ }^{12}$ which enforces the TVD/TVB condition in the mean sense by controlling the reconstructed slopes. This idea was generalized into the form of higher-order moments, ${ }^{13-16}$ and some improved versions have recently been studied as well. ${ }^{17,18}$ On the other hand, limiting strategies based on WENO-type reconstruction were also combined with the higher-order DG method. ${ }^{19-21}$ While most of these limiting studies are formulated in the DG framework, it is possible to apply other higher-order methods. At the same time, it has been noted that the fundamental issue of the limiting algorithms essentially lies in the the mathematical analyses based on the one-dimensional convection equation, which do not in general guarantee the multi-dimensional monotonicity. ${ }^{22,23}$

Especially, the diminished numerical viscosity triggers multi-dimensional oscillations more often, and thus the study on the robust, accurate and efficient shock-capturing philosophy for multi-dimensional flows has become one of the essential issues in higher-order CFD methods. Recently, the multi-dimensional limiting process (MLP) has been successfully proposed in the FVM framework. Compared with traditional limiting strategies, such as the TVD or ENO-type limiting, the MLP limiting can efficiently control unwanted oscillations particularly in multi-dimensional flow situations. By imposing the MLP condition on both cellaveraged and cell-vertex values, the MLP limiting can efficiently follow the multi-dimensional flow physics. A series of previous researches ${ }^{22-25}$ demonstrated that the MLP limiting possesses superior characteristics in terms of accuracy, robustness and efficiency in inviscid and viscous computations on structured and unstructured grids within the FVM framework. Since the proposed limiting algorithm relies only on the MLP stencil regardless of the order of approximation, it facilitates an easy extension to popular higherorder methods, such as DG method. As a way to stabilize the higher-order DG method, the original MLP condition is modified to take into account the behavior of local extrema produced by a cell-wise higherorder approximation. As a consequence, the augmented MLP condition and the MLP-based troubled-cell marker are obtained, which pave the way to obtain the hierarchical DG-MLP formulation. ${ }^{26}$ By examining the numerical Gibbs' phenomenon caused by the sub-cell distribution and its propagation, the $P 1$-projected MLP condition is newly obtained and applied to CPR method within the hierarchical DG-MLP formulation. ${ }^{27}$

In this study, we present the progress of the MLP limiting strategy for higher-order schemes and discuss some issues to treat high-Reynolds number flows around realistic configurations. The paper is organized as follows. The baseline discretization methods are briefly summarized in Section II. Then, the hierarchical MLP limiting with the augmented MLP condition and P1-projected MLP condition is described in Section III. In Section IV, extensive numerical experiments are carried out to assess the performance of the proposed limiting strategy for inviscid and viscous compressible flows involving shocks. Issues for further improvements are discussed in Section V. Finally, conclusions are given in Section VI.

\section{Higher-order CFD Methods}

\section{A. Discontinuous Galerkin Methods}

In order to analyze the inviscid and viscous compressible flows, the Euler and Navier-Stokes equations are considered.

$$
\frac{\partial \mathbf{Q}}{\partial t}+\nabla \cdot\left(\mathbf{F}_{c}-\mathbf{F}_{v}\right)=\mathbf{0}
$$

where $\mathbf{Q}$ is the conservative variable vector, $\mathbf{F}_{c}$ and $\mathbf{F}_{v}$ are the inviscid and viscous flux, respectively.

Discretization using the DG method starts from the weak form of Eq. (1) on the cell $T_{j}$. 


$$
\int_{T_{j}} \frac{\partial \mathbf{Q}}{\partial t} \mathbf{W} d V+\int_{\partial T_{j}}\left(\mathbf{F}_{c}-\mathbf{F}_{v}\right) \cdot \mathbf{n} \mathbf{W} d S-\int_{T_{j}}\left(\mathbf{F}_{c}-\mathbf{F}_{v}\right) \nabla \mathbf{W} d V=\mathbf{0}
$$

where $\mathbf{W}$ is a test function vector, and $\mathbf{n}$ is the outward unit normal vector.

Distribution within a cell is then approximated by the sum of shape functions in a suitably smooth function space $V^{n}$, which usually consists of polynomials of order up to $n$. The test function is also approximated in the same function space $V^{n}$.

$$
\mathbf{Q}_{j}^{h}(\mathbf{x}, t)=\sum_{i=1}^{n d o f} \mathbf{Q}_{j}^{(i)}(t) b_{j}^{(i)}(\mathbf{x}),
$$

where $\mathbf{Q}_{j}^{h}$ is an approximated state variable vector on the cell $T_{j}$ and $b_{j}^{(i)}$ is a shape function. In this computation, all elements are linearly mapped to standard right triangle and we apply local orthogonal shape function by tensor products of Jacobi polynomials. ${ }^{28}$

After applying suitable numerical fluxes, the approximated solution in $V^{n}$ on the cell $T_{j}$ can be written as follows.

$$
\begin{array}{r}
\int_{T_{j}} \frac{\partial \mathbf{Q}_{j}^{h}}{\partial t} \mathbf{B}_{j} d V+\int_{\partial T_{j}} \mathbf{H}_{c}\left(\mathbf{Q}_{j k}^{h}, \mathbf{Q}_{k j}^{h}\right) \cdot \mathbf{n B}_{j} d S-\int_{T_{j}} \mathbf{F}_{c}\left(\mathbf{Q}_{j}^{h}\right) \cdot \nabla_{h} \mathbf{B}_{j} d V \\
-\int_{\partial T_{j}} \mathbf{H}_{v}\left(\mathbf{Q}_{j k}^{h}, \mathbf{\Theta}_{j k}^{h}, \mathbf{Q}_{k j}^{h}, \mathbf{\Theta}_{k j}^{h}\right)+\int_{T_{j}} \mathbf{F}_{v}\left(\mathbf{Q}_{j}^{h}, \mathbf{\Theta}_{j}^{h}\right) \cdot \nabla_{h} \mathbf{B}_{j} d V=\mathbf{0},
\end{array}
$$

where $\mathbf{Q}_{j k}^{h}$ is the cell interface state vector in the direction from $T_{j}$ to its neighboring cell $T_{k}$, and $\mathbf{B}_{j}$ is the vector of the basis functions. $\mathbf{H}_{c}\left(\mathbf{Q}_{L}, \mathbf{Q}_{R}\right)$ and $\mathbf{H}_{v}\left(\mathbf{Q}_{L}, \boldsymbol{\Theta}_{L}, \mathbf{Q}_{R}, \boldsymbol{\Theta}_{R}\right)$ are the tensors of a numerical inviscid and viscous flux function, respectively.

The viscous stress and heat flux are treated with an auxiliary variable vector,

$$
\Theta-\nabla \mathbf{Q}=\mathbf{0}
$$

From the BR2 scheme,${ }^{29}$ Eq. (5) can be discretized by introducing the lift operator.

$$
\Theta_{j k}^{h}=\nabla_{h} \mathbf{Q}_{j k}^{h}+\eta \mathbf{r}_{e}\left(\left[\mathbf{Q}^{h}\right]\right), \quad \Theta_{j}^{h}=\nabla_{h} \mathbf{Q}_{j}^{h}+\mathbf{r}\left(\left[\mathbf{Q}^{h}\right]\right) .
$$

Plugging Eq. (6) into Eq. (4), the primal formulation of the governing equations can be obtained. The detailed derivation to treat the diffusion flux can be founded in Ref. (29). The boundary and domain integration can be performed by the exact formulation or numerical integration with polynomials of order of up to $2 n$ and $2 n+1$, respectively. The present computations adopt the Keast's quadrature rule for triangle and tetrahedron. ${ }^{30}$

\section{B. Correction Procedure via Reconstruction}

The spatial discretization of CPR also starts from the weak form of Eq. (1) on the cell $T_{j}$. After applying monotone numerical fluxes and integrating by part again, the approximated solution in $V^{n}$ on the cell $T_{j}$ can be written as follows.

$$
\int_{T_{j}} \frac{\partial \mathbf{Q}_{j}^{h}}{\partial t} \mathbf{W} d V+\int_{\partial T_{j}}\left(\mathbf{H}\left(\mathbf{Q}_{j k}^{h}, \mathbf{Q}_{k j}^{h}\right)-\mathbf{F}\left(\mathbf{Q}_{j k}^{h}\right)\right) \cdot \mathbf{n} \mathbf{W} d S+\int_{T_{j}} \nabla_{h} \cdot \mathbf{F}\left(\mathbf{Q}_{j}^{h}\right) \mathbf{W} d V=\mathbf{0},
$$

where $\mathbf{Q}_{j k}^{h}$ is the cell interface state vector in the direction from $T_{j}$ to its neighboring cell $T_{k}$, and $\mathbf{H}\left(\mathbf{Q}_{L}, \mathbf{Q}_{R}\right)$ is the tensors of a numerical flux function.

Using the lifting operator, the second integral term can be replaced by a correction field $\delta_{j} \in V^{n}$ and we can obtain following formulation.

$$
\int_{T_{j}}\left[\frac{\partial \mathbf{Q}_{j}^{h}}{\partial t}+\nabla_{h} \cdot \mathbf{F}\left(\mathbf{Q}_{j}^{h}\right)+\delta_{j}\right] \mathbf{W} d V=\mathbf{0} .
$$


By projecting the second term onto $V^{n}$, Eq. (8) with a proper test space can be simplified as follows.

$$
\frac{\partial \mathbf{Q}_{j}^{h}}{\partial t}+\Pi\left(\nabla_{h} \cdot \mathbf{F}\left(\mathbf{Q}_{j}^{h}\right)\right)+\delta_{j}=\mathbf{0},
$$

where $\Pi$ is the projection operator. For the linear conservation law, the projection operator can be skipped. In CPR method, higher-order approximation on each cell is approximated by solution points as

$$
\mathbf{Q}_{j}^{h}(\mathbf{x}, t)=\sum_{i}^{n d o f} \mathbf{Q}_{i, j}^{h}(t) L_{i, j}(\mathbf{x})
$$

where $L_{i, j}(\mathbf{x})$ is the Lagrange polynomial and $\mathbf{Q}_{i, j}^{h}$ is the state vector at solution point $\mathbf{x}_{i}$ on the cell $T_{j}$. We use $\alpha$-optimized node as a solution point for triangular and tetrahedral element. For each solution point, Eq. (9) is imposed as

$$
\begin{array}{r}
\frac{\partial \mathbf{Q}_{i, j}^{h}}{\partial t}+\Pi_{i}\left(\nabla_{h} \cdot \mathbf{F}\left(\mathbf{Q}_{j}^{h}\right)\right)+\delta_{i, j}=0, \\
\delta_{i, j}=\frac{1}{\left|T_{j}\right|} \sum_{f=e_{j k} \in T_{j}} \sum_{l} \alpha_{l, f, j}[\mathbf{F}]_{l, f} \cdot \mathbf{n}\left|e_{j k}\right|,
\end{array}
$$

where

$$
[\mathbf{F}]_{l, f}=\mathbf{H}\left(\mathbf{Q}_{l, j k}^{h}, \mathbf{Q}_{l, k j}^{h}\right)-\mathbf{F}\left(\mathbf{Q}_{l, j k}^{h}\right) .
$$

Here, $\mathbf{Q}_{l, j k}^{h}$ is the approximated value at the $l$-th solution point on the face $e_{j k}$. The details of CPR, such as solution points and $\alpha_{l, f, j}$, can be found in Ref. (7).

To handle the second term in Eq. (11) for non-linear flux, there are two approaches; Lagrange polynomial (LP) and Chain Rule (CR). Both become problematic in solving non-linear equations, such as aliasing error for LP and conservation error for CR. Recently, an efficient cure for the conservation issue was devised ${ }^{31}$ and the present development is based on the conservative CPR method.

The viscous stress and heat flux are treated with an auxiliary variable vector,

$$
\Theta-\nabla \mathbf{Q}=\mathbf{0} .
$$

It is also discretized in CPR method.

$$
\Theta_{i, j}=\left(\nabla \mathbf{Q}_{i, j}^{h}\right)+\frac{1}{\left|T_{j}\right|} \sum_{f=e_{j k} \in T_{j}} \sum_{l} \alpha_{l, f, j}\left[\left(\mathbf{Q}_{l, j k}^{c o m}-\mathbf{Q}_{l, j k}^{h}\right]_{l, f} \mathbf{n}\left|e_{j k}\right| .\right.
$$

Some viscous flux schemes for DG method can be applied to CPR as well, ${ }^{32}$ and the BR2 scheme ${ }^{29}$ is used in this work.

$$
\begin{aligned}
\mathbf{Q}_{l, j k}^{c o m} & =\frac{1}{2}\left(\mathbf{Q}_{l, j k}^{h}+\mathbf{Q}_{l, k j}^{h},\right) \\
\nabla \mathbf{Q}_{l, j k}^{c o m} & =\frac{1}{2}\left(\nabla \mathbf{Q}_{l, j k}^{h}+\mathbf{r}_{l, j k}+\nabla \mathbf{Q}_{l, k j}^{h}+\mathbf{r}_{l, k j}\right) .
\end{aligned}
$$

Here, $\mathbf{r}_{l, j k}$ reflects the jump of the conservative variable at the face $e_{j k}$.

$$
\mathbf{r}_{l, j k}=\sum_{l} \alpha_{l, f, j}\left[\left(\mathbf{Q}_{l, j k}^{c o m}-\mathbf{Q}_{l, j k}^{h}\right]_{l, f} \mathbf{n}\left|e_{j k}\right| .\right.
$$

\section{Unsteady Computation}

For time integration, nonlinear stable methods are applied, such as the third-order accurate TVD RungeKutta method or the five-stage fourth-order accurate strong stability preserving Runge-Kutta method $(\operatorname{SSPRK}(5,4)) .{ }^{33}$ The following definition of time step is used.

$$
\Delta t=\frac{C F L}{2 n+1} \frac{h}{\left|\lambda_{c}^{\max }\right|+d\left|\lambda_{v}^{\max }\right| \frac{2 n+1}{h}},
$$

where $h$ is the radius of the inscribed circle for triangular element. $\lambda_{c}^{\max }$ and $\lambda_{v}^{\max }$ are the maximum wave speed of inviscid and viscous flux, respectively. CFL number has been set to 0.9 for DG/CPR- $P 2$ with third-order TVD Runge-Kutta method, and 1.4 for DG/CPR-P3 with $\operatorname{SSPRK}(5,4)$. 


\section{Higher-order Multi-dimensional Limiting Strategy}

As well as stable time integration methods, an oscillation-control mechanism is essential to resolve compressible flows, especially the flows involved with shock waves. Limiting should be activated only on the troubled-cells to maintain higher-order accuracy across smooth extrema. An accurate troubled-cell marker, followed by a sophisticated limiting, is thus crucial to obtain an accurate monotone profile in the higherorder approximation. Some troubled-cell markers, such as the TVB marker ${ }^{12}$ or KXRCF marker, ${ }^{34}$ have been developed and combined with slope limiters or WENO-type limiters, but the accurate detection of the troubled-cells is not an easy task. Keeping this in mind, we establish the hierarchical MLP limiting strategy for the higher-order CFD methods to detect the troubled-cells. At first, we briefly summarize the MLP-u slope limiters and propose two troubled-cell detecting conditions: the augmented MLP condition and the newly developed $P 1$-projected MLP condition. By combining one of the two conditions with the MLP-based smooth extrema detector, we describes the implementation of the hierarchical MLP limiting algorithm.

\section{A. MLP-u Slope Limiter}

In order to enforce the multi-dimensional monotonicity, the MLP condition has been proposed in the finite volume framework. This condition is simply an extension of the one-dimensional monotonicity condition by considering the case where the direction of local flow gradient is not aligned to the local grid line. The starting point of the MLP condition is that local extrema always occur at vertex point when sub-cell distribution is linear. This observation manifests that, i) treatment of vertex point is essential in limiting stage, ii) all information around vertex point should be incorporated to avoid multi-dimensional oscillations. As a result, we have the following MLP condition.

$$
\bar{q}_{v_{i}}^{\min } \leq q\left(\mathbf{x}_{v_{i}}\right) \leq \bar{q}_{v_{i}}^{\max }
$$

with

$$
\bar{q}_{v_{i}}^{\min }=\min _{T_{k} \in S_{v_{i}}}\left(\bar{q}_{k}\right), \quad \bar{q}_{v_{i}}^{\max }=\max _{T_{k} \in S_{v_{i}}}\left(\bar{q}_{k}\right) .
$$

Here, $\bar{q}_{k}$ is the cell-averaged value on $T_{k}$ and $\mathbf{x}_{v_{i}}$ is the position vector at the vertex $v_{i}$. Two stencils $S_{v_{i}}$ and $S_{T_{j}}$ are defined by

$$
S_{v_{i}}=\left\{T_{k} \mid v_{i} \in T_{k} \text { for some } v_{i} \in T_{j}\right\}, \quad S_{T_{j}}=\left\{T_{k} \mid v_{i} \in T_{k}, \text { for all } v_{i} \in T_{j}\right\} .
$$

In other words, $S_{v_{i}}$ is the union of computational cells sharing the vertex $v_{i}$ and $S_{T_{j}}$ is the union of computational cells sharing any vertex of the cell $T_{j} . S_{T_{j}}$ is called the MLP stencil. ${ }^{24,25}$ It is noted that the MLP condition can be applied to any type of mesh since it does not assume particular mesh connectivity. At the same time, it is also observed that well-controlled vertex value at interpolation/limiting stage makes it possible to produce a monotonic distribution of cell-averaged values. Extensive numerical experiments ${ }^{22-25}$ strongly support that the full realization of Eq. (20) is quite effective to preserve accurate monotone profiles.

This philosophy can be readily extended on unstructured grids with second-order accurate reconstruction. Sub-cell interpolation may start from the unstructured version of the MUSCL-type linear reconstruction as follows.

$$
q_{j}(\mathbf{x})=\bar{q}_{j}+\phi_{j} \nabla \bar{q}_{j} \cdot\left(\mathbf{x}-\overline{\mathbf{x}}_{j}\right)
$$

where $q$ is the the state variable, $\nabla \bar{q}_{j}$ is the gradient within the cell $T_{j}$. $\mathbf{x}$ is the position vector and $\overline{\mathbf{x}}_{j}$ is the the centroid of the cell $T_{j}$. After applying the MLP condition to vertex point, the MLP slope limiter is introduced to ensure multi-dimensional monotonicity by considering all the distributions around the common vertex $v_{i}$. The range of the MLP slope limiting is then obtained as follows.

$$
0 \leq \phi_{j} \leq \max \left(\frac{\bar{q}_{v_{i}}^{\min }-\bar{q}_{j}}{\nabla \bar{q} \cdot\left(\mathbf{x}_{v_{i}}-\overline{\mathbf{x}}_{j}\right)}, \frac{\bar{q}_{v_{i}}^{\max }-\bar{q}_{j}}{\nabla \bar{q} \cdot\left(\mathbf{x}_{v_{i}}-\overline{\mathbf{x}}_{j}\right)}\right)
$$

From Eq. (24), the MLP-u slope limiters can be obtained as follows.

$$
\phi_{M L P}=\min _{\forall v_{i} \in T_{j}}\left\{\begin{array}{ll}
\Phi\left(r_{v_{i}, j}\right) & \text { if } \nabla \bar{q}_{j} \cdot \mathbf{r}_{v_{i}, j} \neq 0 \\
1 & \text { otherwise }
\end{array},\right.
$$


where

$$
r_{v_{i}, j}=\max \left(\frac{\bar{q}_{v_{i}}^{\min }-\bar{q}_{j}}{\nabla \bar{q}_{j} \cdot\left(\mathbf{x}_{v_{i}}-\overline{\mathbf{x}}_{j}\right)}, \frac{\bar{q}_{v_{i}}^{\max }-\bar{q}_{j}}{\nabla \bar{q}_{j} \cdot\left(\mathbf{x}_{v_{i}}-\overline{\mathbf{x}}_{j}\right)}\right) .
$$

By determining $\Phi(r)$ to satisfy the maximum principle, we have the MLP-u1 and MLP-u2 limiters. Detailed implementation in unsteady and steady flows can be found in Refs. $(24,25)$.

The stability characteristics of the MLP limiting is supported by the maximum principle. For multidimensional scalar conservation law, the MLP limiting under linear reconstruction guarantees the following local maximum principle under a suitable CFL condition. ${ }^{24,25}$

$$
\text { If } \min _{T_{k} \in S_{T_{j}}}\left(\bar{q}_{k}^{n}\right) \leq \bar{q}_{j}^{n} \leq \max _{T_{k} \in S_{T_{j}}}\left(\bar{q}_{k}^{n}\right) \text {, then } \min _{T_{k} \in S_{T_{j}}}\left(\bar{q}_{k}^{n}\right) \leq \bar{q}_{j}^{n+1} \leq \max _{T_{k} \in S_{T_{j}}}\left(\bar{q}_{k}^{n}\right) \text {. }
$$

In conjunction with the MLP condition (Eq. (20)), Eq. (27) simply states that the MLP limiting satisfies the MLP condition in the cell-average sense at every time step.

The MLP condition on the MLP stencil makes it realizable to capture multi-dimensional flow physics accurately while maintaining the second-order accuracy in smooth reagion. From Eqs. (20) and (27), the updated solution by the MLP limiting satisfies the maximum principle both on cell-averaged and cell-vertex values, though the stencil involved is a bit different. Thus, the MLP limiting satisfies the LED condition in a truly multi-dimensional way. ${ }^{25}$

\section{B. Augmented MLP Condition}

The MLP condition was used to identify and control the maximum-principle-violating cells in second-order finite volume methods. ${ }^{24,25}$ For higher-order approximation greater than P1 polynomial, additional condition is essential because local extrema no longer appear at vertex point.

If we assume a discontinuity near the vertex point $v_{i}$, as shown in Fig. 1, higher-order $P n$ approximation would trigger unwanted oscillations in the blue-shaded region. For P1 approximation, sub-cell value at any quadrature point in $T_{j}$ can be readily controlled by limiting the vertex value at which local extrema always appear. For greater than $P 1$ approximation, we may have a quadrature point at which sub-cell value does lie outside the range imposed by the MLP condition (Eq. (20)), and thus the maximum principle (Eq. (27)) can be violated. This may occur even if the vertex value does satisfy the MLP condition. Therefore, the MLP condition imposed on a single cell $T_{j}$ is not complete enough to handle higher-order distribution, and it may allow spurious oscillations for the situation depicted in Fig. 1. As a remedy, we require all the approximated vertex values of $S_{v_{i}}$ to satisfy the MLP condition since all $T_{k} \in S_{v_{i}}$ wll be eventually influenced by the presence of the the discontinuity through the dynamic exchange of the cell-interface flexes. In other words, when we check whether $T_{j}$ is a troubled cell or not, we impose the MLP condition on $S_{v_{i}}$ not just on $T_{j}$. From this perspective, we impose a stricter constraint than the MLP condition. The augmented MLP condition is then used as the MLP-based troubled-cell marker for higher-order approximation.

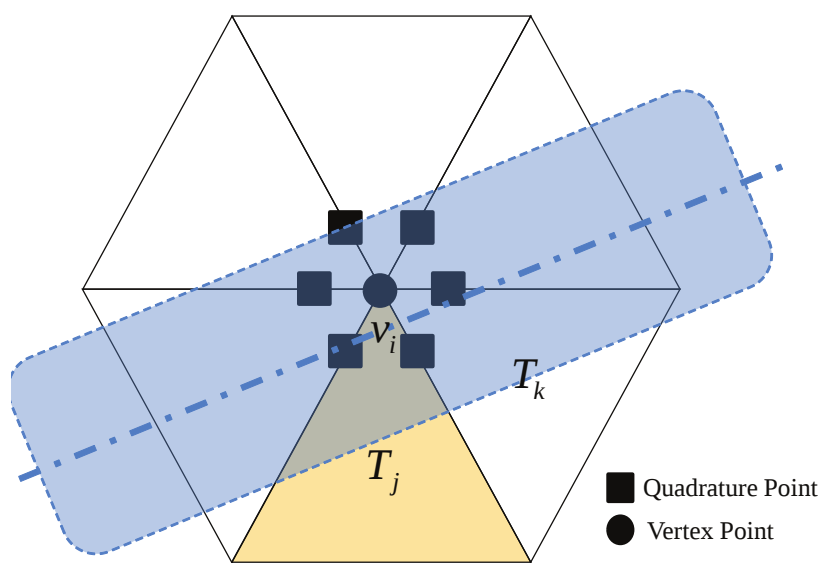

Figure 1: Discontinuity on the MLP stencil. (Dash-dot line is a discontinuity and spurious oscillations may occur in the blue-shaded region.) 


$$
\bar{q}_{v_{i}}^{\min } \leq \min _{T_{k} \in S_{v_{i}}}\left(q_{k}^{h}\left(\mathbf{x}_{v_{i}}\right)\right), \max _{T_{k} \in S_{v_{i}}}\left(q_{k}^{h}\left(\mathbf{x}_{v_{i}}\right)\right) \leq \bar{q}_{v_{i}}^{\max } .
$$

Here, $q_{k}^{h}\left(\mathbf{x}_{v_{i}}\right)$ indicates the approximated value at the vertex $v_{i}$ for $T_{k} \in S_{v_{i}}$. If any $q_{k}^{h}\left(\mathbf{x}_{v_{i}}\right)$ violates Eq.(28), $T_{j}$ is tagged as a troubled cell. Numerical experiments strongly support that the MLP slope limiter with the A-MLP condition is quite successful in handling multi-dimensional oscillations. ${ }^{26}$

\section{P1-projected MLP Condition}

While the augmented MLP condition is successful in detecting multi-dimensional discontinuous profile for higher-order methods, its performance to distinguish normal cells can be further improved. A MLP-based troubled-cell marker is newly proposed by examining the sub-cell distribution of the numerical Gibbs phenomenon. For the purpose of convenience, the analysis is performed using the modal DG method, but its consequence is equally valid to the CPR method.

In order to analyze the numerical behavior across discontinuous profiles, we consider the one-dimensional scalar conservation law with a simple discontinuous initial data as follows.

$$
q_{t}+q_{x}=0, \quad q_{0}(x)= \begin{cases}1 & 0.25<x<0.75 \\ 0 & \text { else }\end{cases}
$$

Computational domain consists of 80 cells on $[0,1]$. Figure 2 shows the sub-cell distribution after a few iterations with DG-P2 and DG- $P 5$ approximation. The black line with the circled ends indicates the $P n$ approximation $\left(q_{j}^{h, P n}(\mathbf{x})\right)$, and the red line denotes cell-averaged value $\left(\bar{q}_{j}\right)$ at each element. The blue line represents the projection of the $P n$ approximation to the linear function space, called the $P$ 1-projected approximation $\left(\Pi^{1} q_{j}^{h, P n}(\mathbf{x})\right)$. From the sub-cell distribution, it is readily noted that oscillations have already developed in the higher-order modes and they are propagating into the cell-averaged values, though the cellaveraged values still maintain a monotonic profile. When oscillations in the higher-order modes are triggered, the $P 1$-projected approximation acts as a precursor to warn non-monotone distributions by developing a steep gradient. Figure 3 shows the sub-cell distribution of the Burgers' equation at $t=0.3$. The computational domain is the same and the initial condition is a sine wave as follows.

$$
q_{0}(x)=\sin (2 \pi x) .
$$

Lines and symbol are the same as in Fig. 2. As shock discontinuity is developed, oscillations start in the higher-order modes and, as a result, steep gradients of the projected- $P 1$ approximations are observed again.

From this observation, it is obtained that, i) oscillations are hierarchically propagating from the higherorder modes into the cell-averaged value, ii) oscillations strongly affect the $P 1$-projected term to produce a monotonicity-violating steep gradient. This indicates that examining the monotonicity of the $P 1$-projected term is enough to detect oscillations in the higher-order modes. Thus, we newly propose a simple and efficient limiting condition for higher-order approximations as follows.

$$
\min \left(\bar{q}_{i}, \bar{q}_{i+1}\right) \leq \Pi^{1} q_{j}^{h, P n}\left(x_{i+1 / 2}\right) \leq \max \left(\bar{q}_{i}, \bar{q}_{i+1}\right) .
$$

It is noted that this condition limits the linear term, and thus it can be readily extended into multiple dimensions by embracing the MLP philosophy on vetrtex point, leading to the following P1-projected MLP condition.

$$
\bar{q}_{v_{i}}^{\min } \leq \Pi^{1} q_{j}^{h, P n}\left(\mathbf{x}_{v_{i}}\right) \leq \bar{q}_{v_{i}}^{\max } .
$$

Figures 4 and 5 show the computed results of the same one-dimensional linear wave equation and Burgers' equation by applying the $P 1$-projected MLP condition at $t=1.0$ and $t=0.3$, respectively. It is noted that the $P 1$-projected MLP condition successfully distinguishes the discontinuous profiles, spurious oscillations are effectively suppressed, and thus the monotonicity of the cell-averaged values are well maintained. Slight non-monotonicity in the higher-order sub-cell distribution may be observed at the head and tail of the discontinuity, but their magnitude is well controlled by the neighboring cell-averaged values. Thus, sub-cell oscillations do not affect the monotonicity of the cell-averaged values. Results on multi-dimensional extension is examined in the next section. 


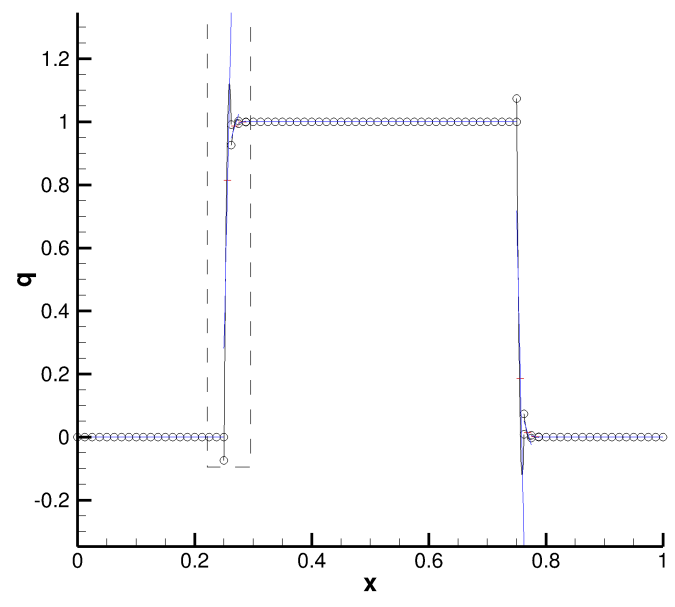

(a) DG-P2

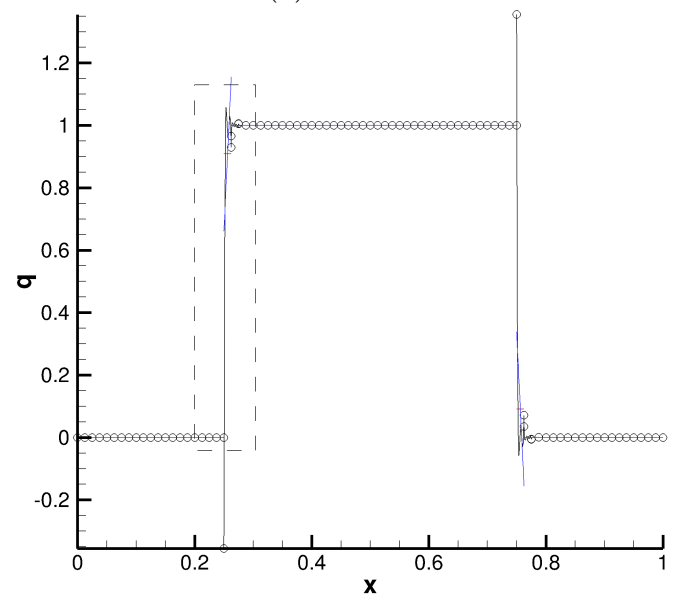

(c) DG-P5



(b) DG-P2, closeup



(d) DG-P5, closeup

Figure 2: Sub-cell distribution of square wave after a few iterations 




(a) DG-P3

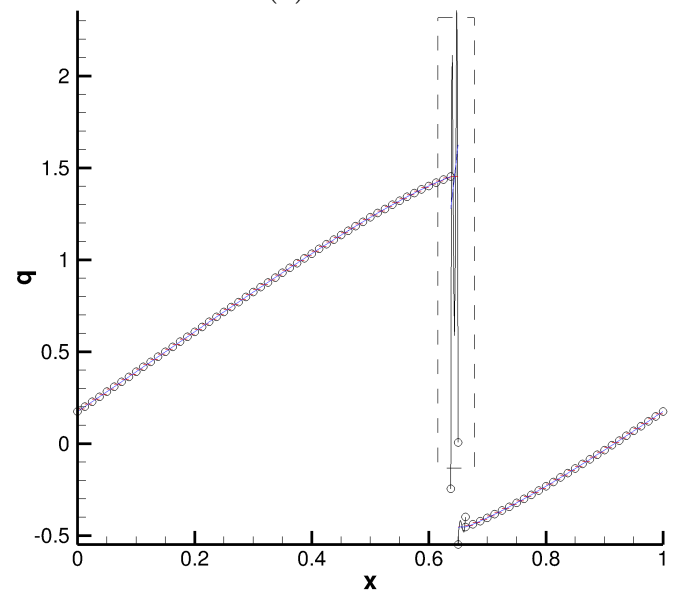

(c) DG-P4



(b) DG-P3, closeup

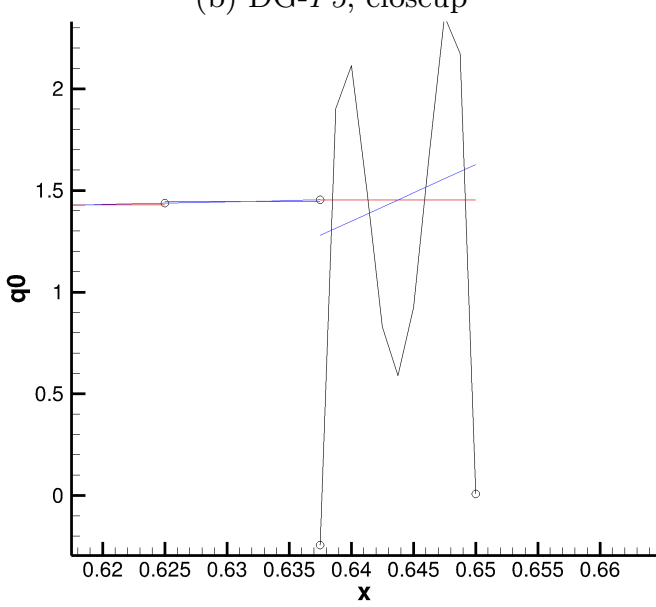

(d) DG- $P$ 4, closeup

Figure 3: Sub-cell distribution of Burgers' equation at $t=0.3$. 


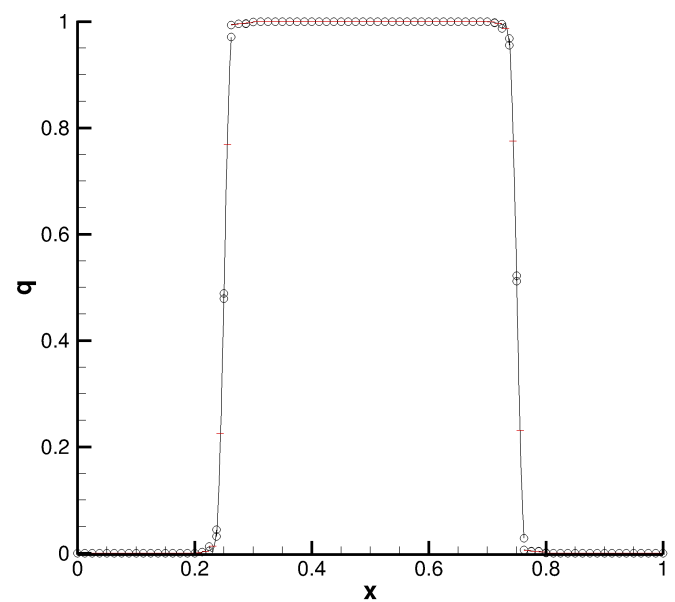

(a) DG-P2

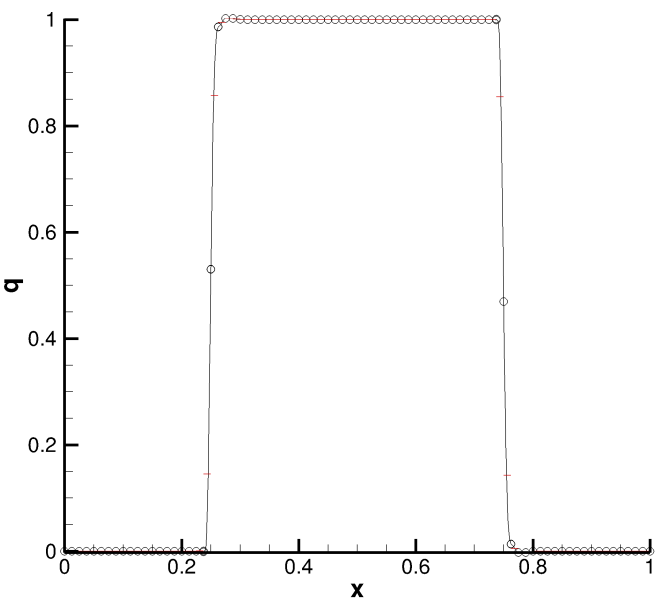

(b) DG-P5

Figure 4: Sub-cell distribution of square wave at $t=1.0$ with $P 1$-projected MLP.

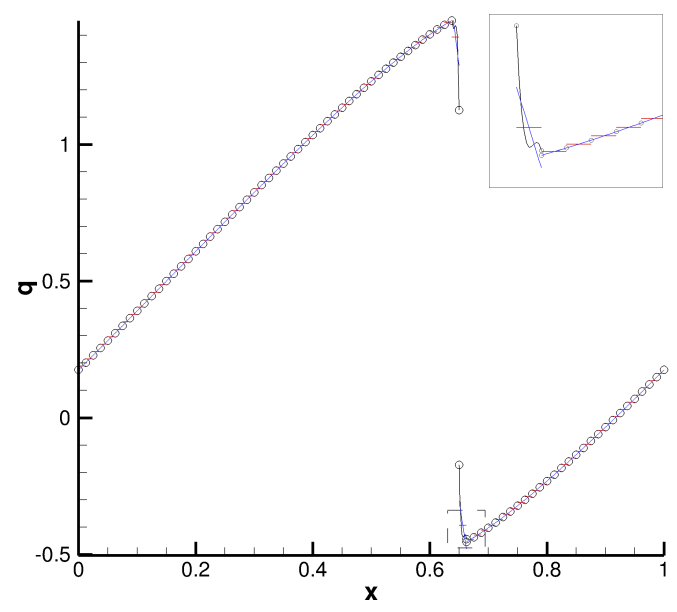

(a) DG-P3

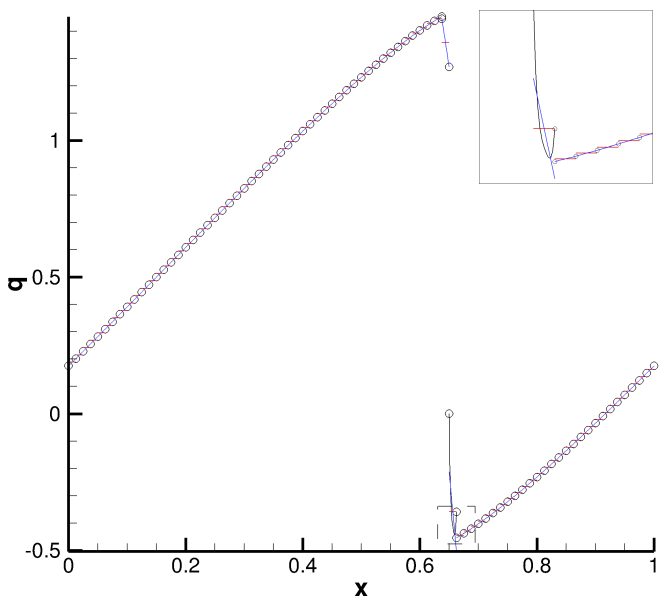

(b) DG-P4

Figure 5: Sub-cell distribution of Burgers' equation at $t=0.3$ with $P 1$-projected MLP.

\section{Hierarchical MLP Limiting Algorithm}

The augmented MLP and P1-projected MLP conditions in themselves do not provide any mechanism to distinguish local smooth extrema. In order to preserve the accuracy across smooth extrema, we introduce a MLP extrema detector by examining the behavior of local extrema around the vertex $v_{i}$ of the cell $T_{j}$.

First, we decompose the $P n$ approximation into the cell-average part, the linear part ( $P n$-projected slope) and the higher-order part ( $P 1$-filtered $P n)$ as follows:

$$
q_{j}^{h, P n}\left(\mathbf{x}_{v_{i}}\right)=\bar{q}_{j}+\underbrace{\left(\Upsilon\left(\mathbf{x}_{v_{i}}\right)-\bar{q}_{j}\right)}_{P n \text {-projected slope }}+\underbrace{\left(q_{j}^{h, P n}\left(\mathbf{x}_{v_{i}}\right)-\Upsilon\left(\mathbf{x}_{v_{i}}\right)\right)}_{P 1 \text {-filtered } P n},
$$

where

$$
\Upsilon\left(\mathbf{x}_{v_{i}}\right)=\Pi^{1} q_{j}^{h, P n}\left(\mathbf{x}_{v_{i}}\right) .
$$

The starting point is that the $P n$-projected slope can be interpreted as the average slope of the $P n$ approximation. Then, from the mean value theorem, if local extrema appear in a small neighborhood of the vertex $v_{i}$, the gradient direction of $q_{j}^{h, P n}\left(\mathbf{x}_{v_{i}}\right)$ could be quite different from the direction of the $P n$-projected 
slope. If one goes up, the other would go down. Even if they show the same trend, the magnitude of the gradient of $q_{j}^{h, P n}\left(\mathbf{x}_{v_{i}}\right)$ would be smaller than that of the $P n$-projected slope. From this observation, we can readily deduce the following condition to detect smooth extrema near the vertex $v_{i}$.

- $\mathbb{C} 1$. If there is a local maximum near the vertex $v_{i}$,

$P n$-projected slope $>0, P 1$-filtered $P n<0$, and $q_{j}^{h, P n}\left(\mathbf{x}_{v_{i}}\right)>\bar{q}_{v_{i}}^{\min }$.

- $\mathbb{C} 2$. If there is a local minimum near the vertex $v_{i}$,

$$
P n \text {-projected slope }<0, P 1 \text {-filtered } P n>0 \text {, and } q_{j}^{h, P n}\left(\mathbf{x}_{v_{i}}\right)<\bar{q}_{v_{i}}^{\max } \text {. }
$$

The last inequalities $\left(q_{j}^{h, P n}\left(\mathbf{x}_{v_{i}}\right)>\bar{q}_{v_{i}}^{\min }, q_{j}^{h, P n}\left(\mathbf{x}_{v_{i}}\right)<\bar{q}_{v_{i}}^{\max }\right)$ are necessary to treat a stiff gradient which may include physical discontinuities. Finally, in order to deal with a nearly constant region particularly when local grids become very coarse, we add the following deactivation threshold.

$$
\left|q_{j}^{h, P n}\left(\mathbf{x}_{v_{i}}\right)-\bar{q}_{j}\right| \leq \max \left(\epsilon \times\left|\bar{q}_{j}\right|,\left|T_{j}\right|\right),
$$

where $\epsilon$ is a small number to distinguish a constant region with machine error, $1 \times 10^{-3}$ is a reasonable choice. $\left|T_{j}\right|$ is the area/volume of the cell $T_{j}$. Most of the extrema are well recognized by the condition $\mathbb{C} 1$ and $\mathbb{C} 2$, and only a very few cells are detected by Eq. (36).

By combining the A-MLP or P1-projected MLP condition (Eq. (28) or Eq. (32)) and the extrema detector (Eq. (35) with Eq. (36)), we formulate the hierarchical MLP limiting strategy for arbitrary Pn approximation. The limiting procedure higher than $P \mathscr{2}$ approximation can be written as follows.

$$
\begin{aligned}
q_{j}^{h, p 2}(\mathbf{x}) & =\bar{q}_{j}+\phi_{M L P}\left(P 1_{j}(\mathbf{x})\right)+\varphi_{j}^{P 2}\left(P 2_{j}(\mathbf{x})\right) \\
q_{j}^{h, P 3}(\mathbf{x}) & =\bar{q}_{j}+\phi_{M L P}\left(P 1_{j}(\mathbf{x})\right)+\varphi_{j}^{P 2}\left(P 2_{j}(\mathbf{x})+\varphi_{j}^{P 3}\left(P 3_{j}(\mathbf{x})\right)\right), \\
\ldots & \\
q_{j}^{h, P n}(\mathbf{x}) & =\bar{q}_{j}+\phi_{M L P}\left(P 1_{j}(\mathbf{x})\right) \\
& +\varphi_{j}^{P 2}\left(P 2_{j}(\mathbf{x})+\varphi_{j}^{P 3}\left(P 3_{j}(\mathbf{x})+\varphi_{j}^{P 4}\left(\ldots+\varphi_{j}^{P n} P n_{j}(\mathbf{x})\right)\right)\right) .
\end{aligned}
$$

Here,

$$
P m_{j}(\mathbf{x})=\Pi^{m} q_{j}^{h, P n}(\mathbf{x})-\Pi^{m-1} q_{j}^{h, P n}(\mathbf{x}) .
$$

$\Pi^{m}$ indicates a projection operator to $P m$ space. $\varphi_{j}^{P n}$ is the hierarchical MLP troubled-cell marker applied to the $P m_{j}$ mode of the cell $T_{j}$ as follows.

$$
\varphi_{j}^{P n}=\min _{\forall v_{i} \in T_{j}}\left(\psi_{v_{i}, j}^{P n}\right)
$$

Depending on the choice of $\varphi_{j}^{P n}$, we can propose two versions of the hierarchical MLP limiting.

- Hierarchical MLP limiting with the augmented MLP condition:

$$
\psi_{v_{i}, j}^{P n}= \begin{cases}1 & \text { if Eq. (28) or Eq. (35) with Eq. (36) is satisfied } \\ 0 & \text { else. }\end{cases}
$$

- Hierarchical MLP limiting with the P1-projected MLP condition:

$$
\psi_{v_{i}, j}^{P n}= \begin{cases}1 & \text { if Eq. (32) or Eq. (35) with Eq. (36) is satisfied } \\ 0 & \text { else. }\end{cases}
$$

The MLP limiting procedure of Eq. (37) is applied in a hierarchical manner from a higher-order $P n_{j}$ mode to the lowest $P 2_{j}$ mode. The implementation procedure can be summarized as follows:

1. Apply the augmented MLP or P1-projected MLP condition (Eq. (28) or Eq. (32)) to the Pn approximated solution at every vertex $v_{i}$ of the cell $T_{j}$. 
2. Compute the hierarchical troubled-cell marker $\varphi_{j}^{P n}$ (Eq. (39)).

3. If the cell $T_{j}$ is tagged as a normal cell (or $\varphi_{j}^{P n}=1$ ), the highest order term is kept unlimited and the limiting procedure is completed. Otherwise $\left(\varphi_{j}^{P n}=0\right)$,

(a) if $n>2$, project the entire polynomial approximation onto $V^{n-1}$ space and obtain $P(n-1)_{j}(\mathbf{x})$. And, repeat Step 2 for $P(n-1)$ approximation.

(b) if $n=2$, project the entire polynomial approximation onto $V^{1}$ space. $P 1_{j}(\mathbf{x})$ is limited by the MLP-u slope limiters (Eq. (25)).

In modal DG with orthogonal basis functions, the projection procedure is realized by simply truncating the higher-order $P n_{j}$ mode. In case of nodal formulation, such as CPR, the projection procedure is carried out by obtaining $q_{j}^{h, P m}(\mathbf{x})=\sum_{l} \tilde{q}_{l, j}^{h, P m} L_{l, j}^{P m}(\mathbf{x})(m<n)$ from the $P n$ approximation as follows.

$$
\sum_{l}\left(\int_{T_{j}} L_{l, j}^{P m}(\mathbf{x}) L_{k, j}^{P m}(\mathbf{x}) d V\right) \tilde{q}_{l, j}^{h, P m}=\int_{T_{j}} q_{j}^{h, P n}(\mathbf{x}) L_{k, j}^{P m}(\mathbf{x}) d V .
$$

Here, $L_{i, j}^{P n}(\mathbf{x})$ are the $n$-th order Lagrange polynomial for the solution point $\mathbf{x}_{i}$ on the cell $T_{j}$ for $P n$ approximation. Using this $L 2$ projection procedure, $\tilde{q}_{i, j}^{h, P m}$ is obtained and the hierarchical MLP is implemented as follows. After checking the MLP troubled-cell marker for $P n$ approximation, the projection operator to $P m$ space is defined as follows.

$$
\Pi^{m} q_{j}^{h, P n}(\mathbf{x})=\sum_{l} \tilde{q}_{l, j}^{h, P m} L_{l, j}^{P m}(\mathbf{x}) .
$$

This procedure is performed in a hierarchical manner, and the MLP-u slope limiter is finally applied to the $P 2_{j}$ mode. Figure 6 shows the schematic summary of the hierarchical MLP limiting procedure for the DG and CPR methods.

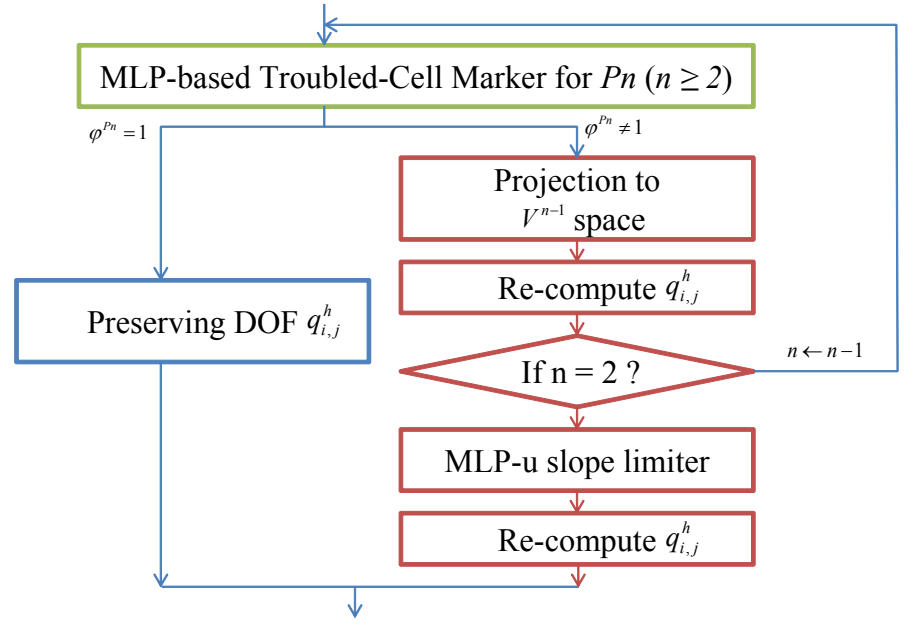

Figure 6: Flowchart of the MLP limiting procedure.

\section{E. Extension to Flow System}

The troubled-cell markers and slope limiters for system of equations should reflect the flow physics. Density or entropy variable is used for the MLP troubled-cell marker to identify physical discontinuities. The marker by entropy variable is slightly more robust, especially resolving very strong shock or nearly vacuum state. In this study, however, all computations are carried out with the MLP troubled-cell marker by density variation. As in the case of FVM, the MLP limiting is applied to conservative variables since characteristic decomposition is not essential. Negative pressure and/or density at solution points, if any, is treated by employing a simple scaling technique. ${ }^{17}$ For efficient computation, grid partitioning by the METIS library ${ }^{35}$ and parallelization with the MPI standard are implemented. 


\section{Numerical Results}

Extensive numerical experiments are carried out to assess the performance of the hierarchical MLP for the DG and CPR methods. Some well-known test problems on 2-D triangular and 3-D tetrahedral grids are examined up to the $P 3$ accuracy. As a numerical flux, RoeM ${ }^{36}$ scheme and AUSMPW+ ${ }^{37}$ scheme are adopted.

\section{A. Convergence Study}

Numerical accuracy in multi-dimensional inviscid compressible flows without shock wave is examined. The initial condition is set to be $\left(\rho_{0}, u_{0}, v_{0}, w_{0}, p_{0}\right)=(1+0.2 \sin (\pi(x+y+z)), 0.5,0.3,0.2,1)$, and the exact solution of density is $1+0.2 \sin (\pi(x+y+z-t))$. The computational domain is $[0,2] \times[0,2] \times[0,2]$, and a periodic boundary condition is applied. Tetrahedral meshes are generated by Gmesh software. ${ }^{38}$ The error between a numerical solution and the exact solution is measured by $L^{p}$ error with $p=\infty, 1$ and 2 norm. For $p \geq 1$,

$$
L^{p} \text { error }=\frac{1}{\sum_{j}\left|T_{j}\right|}\left(\sum_{j}\left|T_{j}\right| \sum_{i}^{n d o f}\left|q_{j}^{h}\left(\mathbf{x}_{i}\right)-q_{\text {exact }}\left(\mathbf{x}_{i}\right)\right|^{p}\right)^{1 / p},
$$

where $\left|T_{j}\right|$ is the area of cell $T_{j} . L^{\infty}$ error is computed using the maximum difference among the solution points. Table 1 shows the result of grid refinement test on tetrahedral grids. While the error of DG is smaller than that of CPR, both methods with MLP maintains the formal order-of-accuracy in smooth region.

Table 1: Grid refinement test for Euler equations on irregular grids at $t=0.25$.

\begin{tabular}{ccccc}
\hline & DOF & $L^{2}$ & Order & CPU Time \\
\hline DG-P2 & $10368 \times 10$ & $3.1261 \mathrm{E}-04$ & - & 11.908369 \\
& $24576 \times 10$ & $1.2678 \mathrm{E}-04$ & 3.14 & 35.902534 \\
& $48000 \times 10$ & $6.5227 \mathrm{E}-05$ & 2.98 & 88.090376 \\
& $82944 \times 10$ & $3.7780 \mathrm{E}-05$ & 3.01 & 183.584061 \\
& $196608 \times 10$ & $1.5875 \mathrm{E}-05$ & 3.00 & 569.611446 \\
\hline DG-P3 & $10368 \times 20$ & $2.3075 \mathrm{E}-05$ & - & 52.193214 \\
& $24576 \times 20$ & $7.1225 \mathrm{E}-06$ & 4.09 & 161.625897 \\
& $48000 \times 20$ & $2.9425 \mathrm{E}-06$ & 3.96 & 403.676706 \\
& $82944 \times 20$ & $1.4046 \mathrm{E}-06$ & 4.06 & 846.423544 \\
& $196608 \times 20$ & $4.4356 \mathrm{E}-07$ & 4.01 & 2627.760445 \\
\hline CPR-P2 & $10368 \times 10$ & $1.5848 \mathrm{E}-03$ & - & 8.663248 \\
& $24576 \times 10$ & $6.1598 \mathrm{E}-04$ & 3.28 & 27.033716 \\
& $48000 \times 10$ & $3.1822 \mathrm{E}-04$ & 2.96 & 63.020937 \\
& $82944 \times 10$ & $1.9257 \mathrm{E}-04$ & 2.75 & 129.660023 \\
& $196608 \times 10$ & $7.9686 \mathrm{E}-05$ & 3.07 & 392.252533 \\
\hline CPR-P3 & $10368 \times 20$ & $1.2068 \mathrm{E}-04$ & - & 29.494923 \\
& $24576 \times 20$ & $3.5364 \mathrm{E}-05$ & 4.27 & 91.234174 \\
& $48000 \times 20$ & $1.5969 \mathrm{E}-05$ & 3.56 & 226.003312 \\
& $82944 \times 20$ & $7.1925 \mathrm{E}-06$ & 4.37 & 474.947794 \\
& $196608 \times 20$ & $2.2515 \mathrm{E}-06$ & 4.04 & 1426.850471 \\
\hline
\end{tabular}

\section{B. Shock Tube Problems}

Some well-known one-dimensional shock tube problems are computed in a three-dimensional manner to examine the capability of resolving various linear and non-linear waves on unstructured grids. 


\section{Sod and Harten-Lax Problems}

This test is to examine the capability to resolve various linear and non-linear waves on unstructured grids. The computational domain is $[0,1] \times[0,0.01] \times[0,0.01]$ with a tetrahedralization of 101 vertices in the $x$-direction and 11 vertices in the $y, z$-direction. Two Riemann-type initial conditions are considered.

Sod problem:

$$
\begin{aligned}
& \left(\rho_{L}, u_{L}, v_{L}, w_{L}, p_{L}\right)=(1,0,0,0,1) \\
& \left(\rho_{R}, u_{R}, v_{R}, w_{R}, p_{R}\right)=(0.125,0,0,0,0.1) .
\end{aligned}
$$

Harten-Lax problem:

$$
\begin{aligned}
& \left(\rho_{L}, u_{L}, v_{L}, w_{L}, p_{L}\right)=(0.445,0.698,0,0,3.528), \\
& \left(\rho_{R}, u_{R}, v_{R}, w_{R}, p_{R}\right)=(0.5,0,0,0,0.571) .
\end{aligned}
$$

The interface is initially located at $x=0.5$ and the RoeM scheme is applied.

Figures 7 show the density distributions along the $x$-axis at $t=0.2$ (the Sod problem) and $t=0.13$ (the Harten-Lax problem), respectively. Discontinuous profiles are successfully captured without spurious oscillations. Compared to the FVM with MLP solutions, the results with the DG-MLP and CPR-MLP methods exhibit a clear advantage in capturing contact discontinuites and expansion corners.

\section{Shock-entropy Wave Interaction (Shu-Osher Problem)}

As another standard benchmark test for high-resolution schemes, the interaction between a shock and an entropy wave ${ }^{39}$ is considered. Similar to the previous cases, the one-dimensional setting is extended into three-dimensional tetrahedral grids. The computational domain is $[-5,5] \times[-0.1,0.1] \times[-0.1,0.1]$ with a tetrahedralization of 301 vertices in the $x$-direction and 7 vertices in the $y, z$-direction. The initial profile consists of a shock and an entropy wave as follows:

$$
\begin{array}{ccl}
\left(\rho_{L}, u_{L}, v_{L}, p_{L}\right) & =(3.857143,2.629369,0,10.333333) & \text { if } x<-4, \\
\left(\rho_{R}, u_{R}, v_{R}, p_{R}\right) & =(1+0.2 \sin (5 x), 0,0,1.0) & \text { else. }
\end{array}
$$

Figure 8 compares the density distributions along the center line by DG and CPR with MLP limiting at $t=1.8$. The reference solution is the one obtained by computing the equivalent one-dimensional Euler equations on 8,000 grid points using the TVD-MUSCL scheme. It is noted again that the MLP limiting, successfully combined with the DG and CPR methods, accurately suppresses unwanted oscillations.

\section{Double Mach Reflection}

This is one of the most well-known test cases for high-resolution schemes. ${ }^{40}$ With the computational domain of a tube with a 30 degree ramp, a strong moving shock with $M_{s}=10$ impinges on the ramp. The RoeM scheme is used as the numerical flux, and computation is carried out until $t=0.2$.

Figure 9 shows the density contours of the DG and CPR methods on triangular grids $(h=1 / 100)$. The proposed limiting methods successfully provide monotonic solutions. The higher-order DG and CPR with MLP methods can significantly improve the resolution of the sheer layer and the vortex which is developed from the shock triple point and the Mach stem. It is also noted that the P1-projected MLP condition turns out to be more accurate in captureing detail flow structure.

\section{Interaction of Shock Wave with 2-D Wedge}

This test case is known as the Scharldin's problem. ${ }^{41}$ As a moving shock strikes a two-dimensional wedge, reflected shock waves and a complex vortex pattern are generated after the wedge end. A regular triangle with the unit length is contained in the $[-2.5,4.6] \times[-2.5,2.5]$ computational domain, and the wedge tip is positioned at the origin. As the initial condition, a moving shock with $M_{s}=1.34$ is located at $x=0$. The downstream state is $\left(\rho_{0}, u_{0}, v_{0}, p_{0}\right)=(1.4,0,0,1.0)$. The RoeM flux scheme is applied.

Figures 10 compares the numerical Schlieren images produced by the FVM, DG and CPR with MLP at $t=3.25$. The computed results confirm again that the higher-order DG and CPR with the MLP provides a resolution sufficient to capture the detailed shock pattern and flow structures. Compared with experimental visualization, ${ }^{42}$ the small vortices produced by the first shock-vortex interaction at the wedge corner are particularly well captured by the hierarchical limiting with the P1-projected MLP condition. 




(a) Sod problem

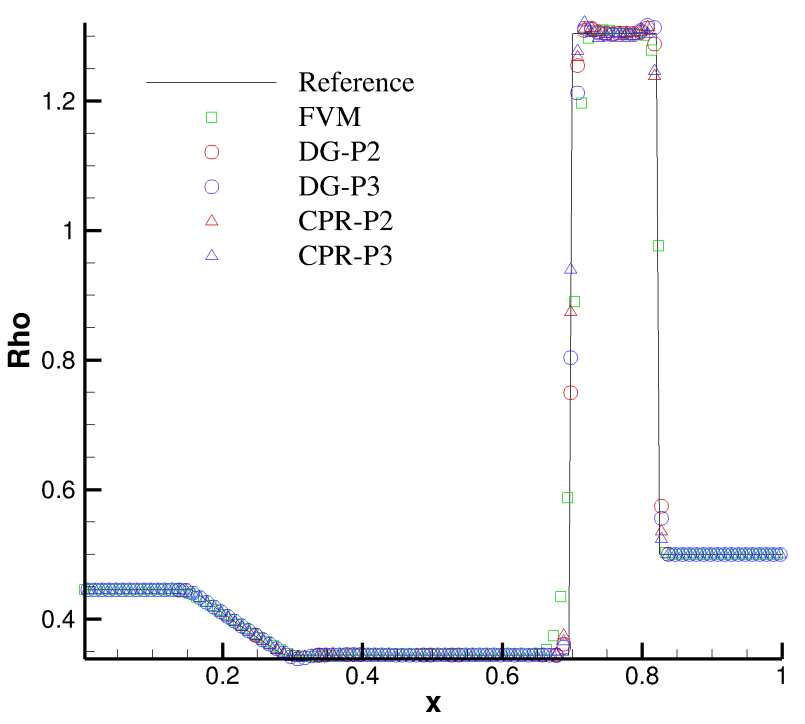

(b) Harten-Lax problem

Figure 7: Density distributions along center line (Shock tube problem).

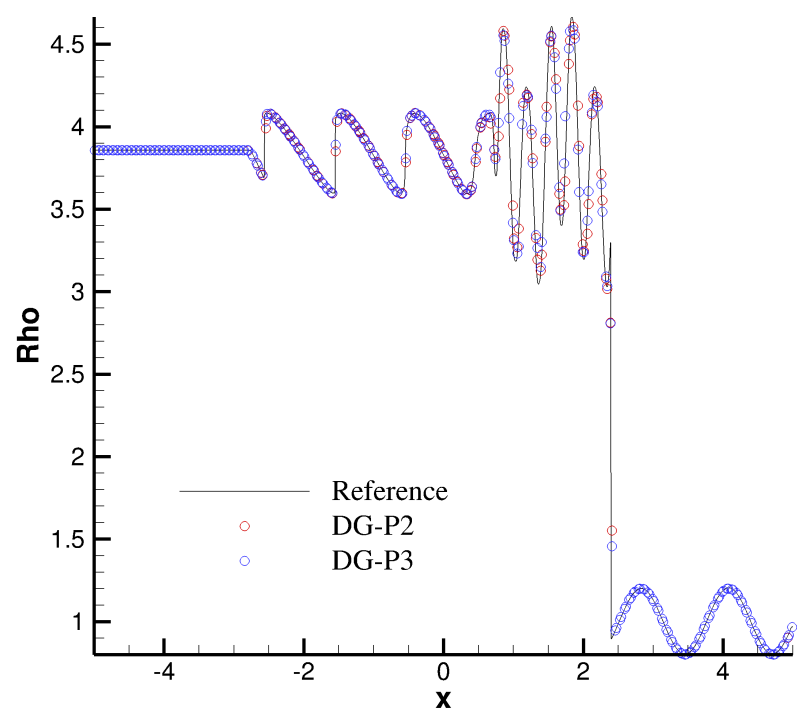

(a) DG-MLP



(b) CPR-MLP

Figure 8: Density distributions along center line (Shock-entropy wave Interaction). 


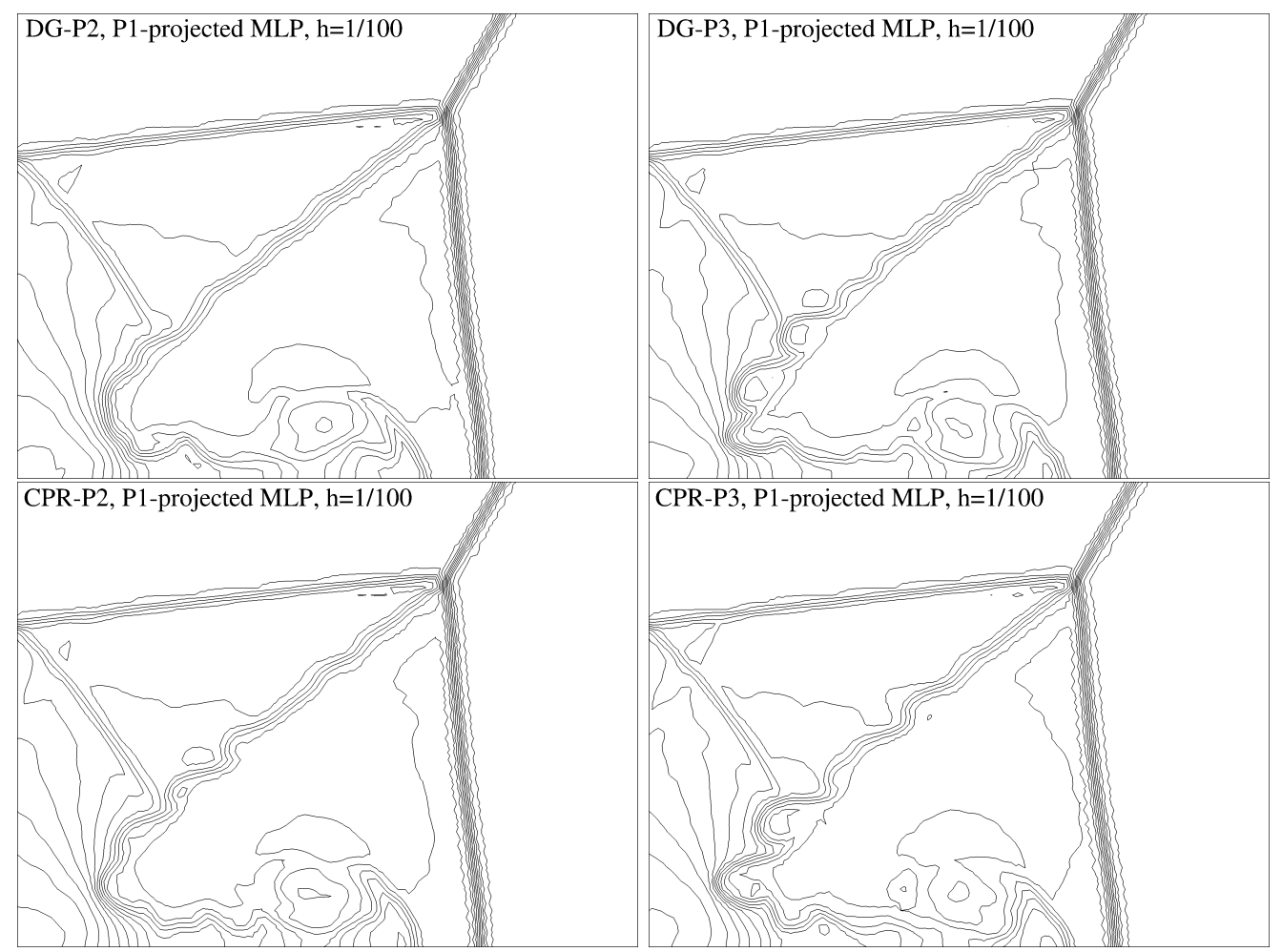

Figure 9: Comparison of density contours around the double Mach stem

\section{E. Interaction of Shock Wave With Density Bubble}

The unsteady flow physics of shock-bubble interactions has been extensively studied because it can provide a numerical testbed for the generation of vorticity and turbulence in compressible flows. ${ }^{43}$ The bubble dynamics, belonging to a two-phase flow field, is simplified with a single-phase gas model by assuming a density difference across the bubble interface. As a moving shock impinges on density bubble, the KelvinHelmholtz instability from the tail of the counterclockwise primary vortex is developed along the bubble interface resulting in complex vortical structure.

Exploiting the flow symmetry, computational domain is a quadrant of shock tube with the interval of $[0,3 L]$ in the $x$-direction and $[0, L] \times[0, L]$ in the $y$ - $z$ plane with $L=4.45$. Initially, the quarter of spherical bubble with $r=2.5$ is placed at $x=3.5$, and the moving shock with $M_{s}=3.0$ is located at $x=0.5$. Grid system consists of 8.90 million tetrahedral elements. With Tachyon 2 supercomputer at KISTI, MPI parallel computation was performed with 1024 CPUs to reach at $t=3.5$.

Figures 11 and 12 shows the three-dimensional density contours by CPR-P3 with the hierarchical MLP limiting with $P 1$-projected MLP condition. The primary and secondary vortex structure caused by the baroclinic torque, the tail vortical structure after the primary vortex, and the 3-D monotonic shock are well captured. Figure 13 compares the density contours on the $x-y$ plane. Higher-order approximation/reconstruction with MLP captures distortion of the primary vortex and the instability around the tail.

\section{F. Oblique Shock Mixing Layer Interaction}

This test is carried out to examine the resolution of small scale vortical structures interacting with a shock discontinuity. ${ }^{44}$ A spatially developing compressible mixing layer produces a series of vortices, and the oblique shock originating from the upper-left corner impinges on the mixing layer. The oblique shock is deflected by the shear layer and then reflects again from the bottom slip wall, leading to the interaction between downstream vortices and the reflected shock. 


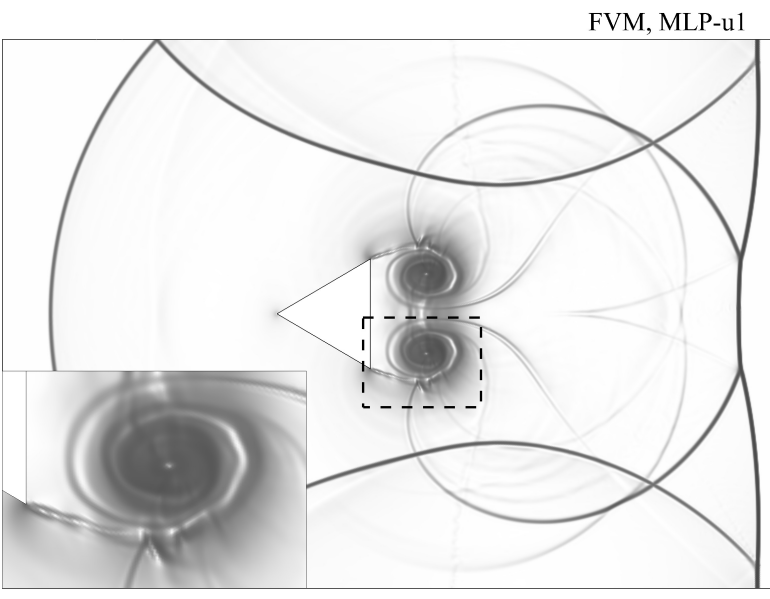

(a) FVM



(c) DG-P2, P1-projected MLP



(e) CPR-P2, P1-projected MLP

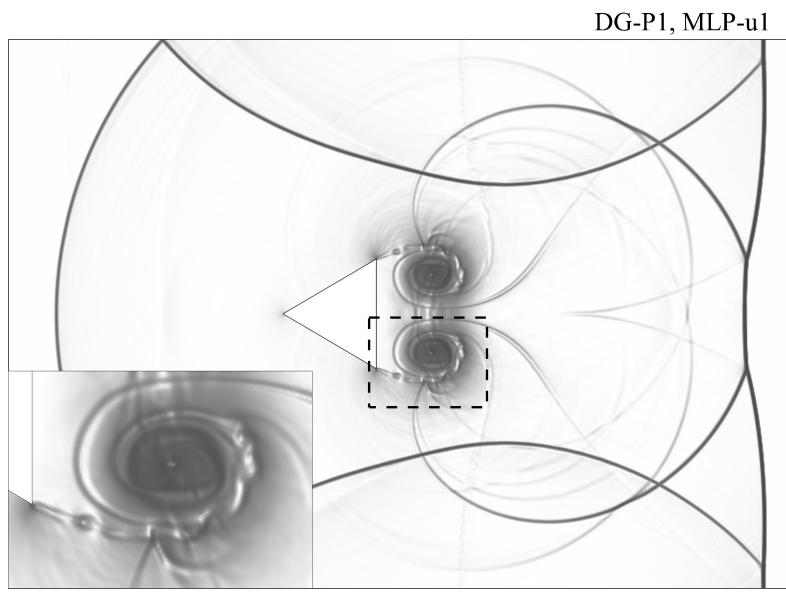

(b) DG-P1

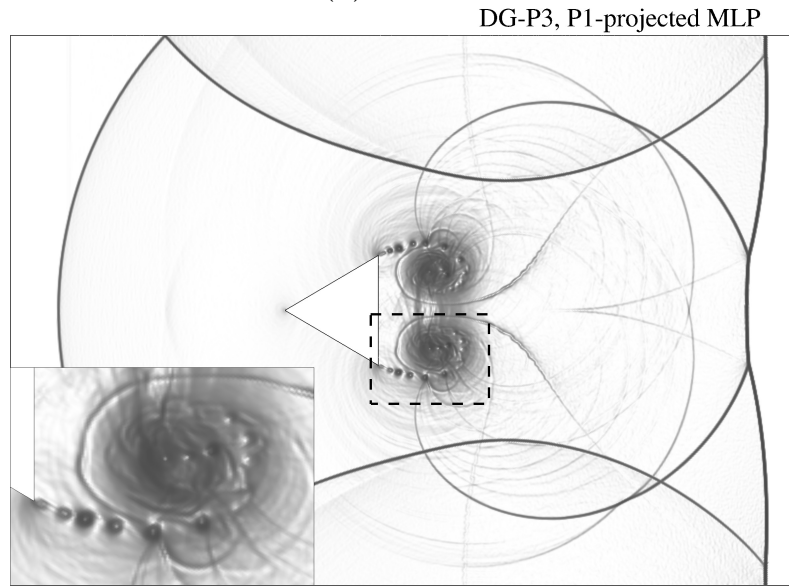

(d) DG-P3, P1-projected MLP



(f) CPR-P3, P1-projected MLP

Figure 10: Comparison of numerical Schlieren images of interaction of shock wave with 2-D wedge at $t=3.25$. (Bottom left corner: Close-up view around the primary vortex) 


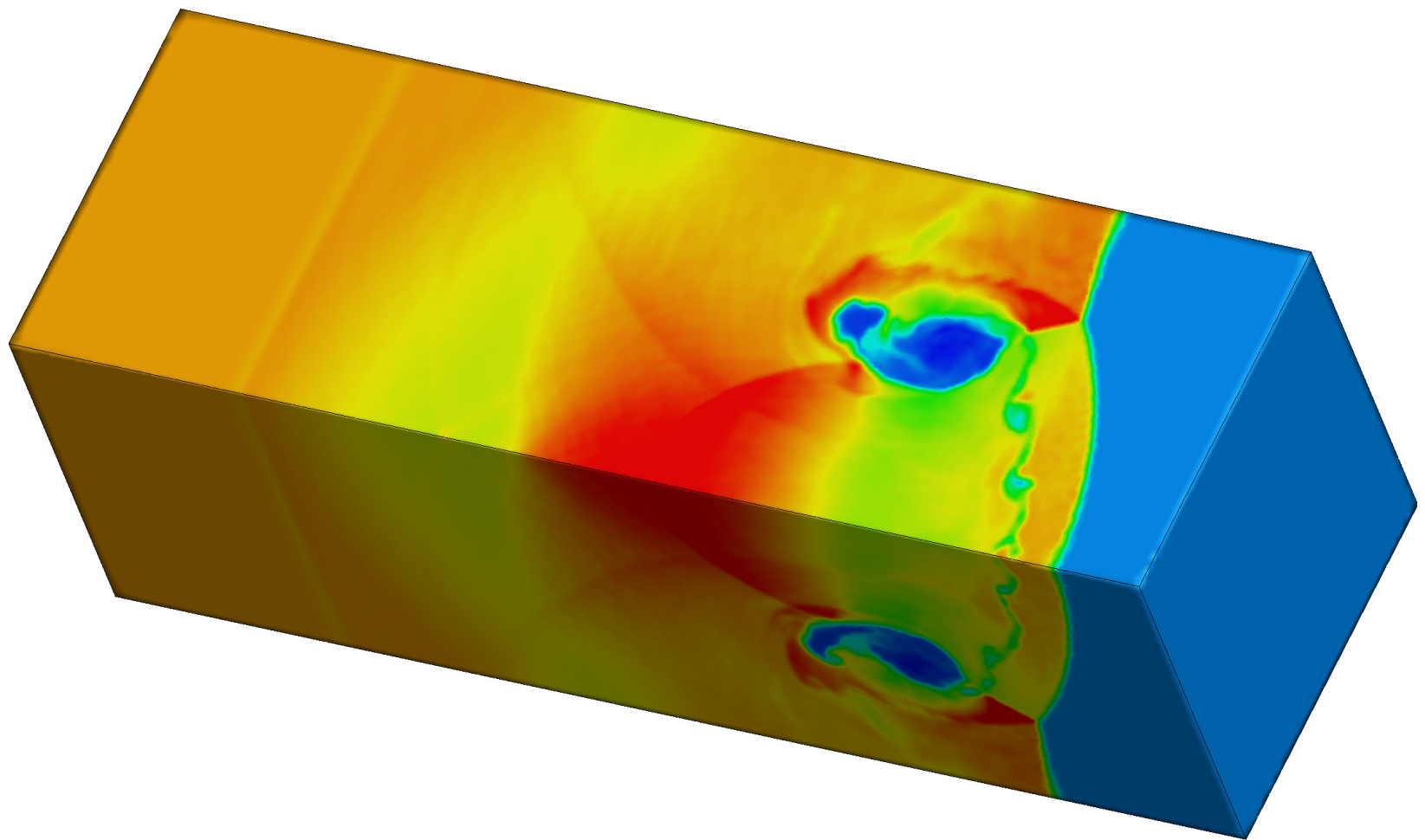

Figure 11: Density contours of interaction of 3-D density bubble with shock wave at $t=3.5$.

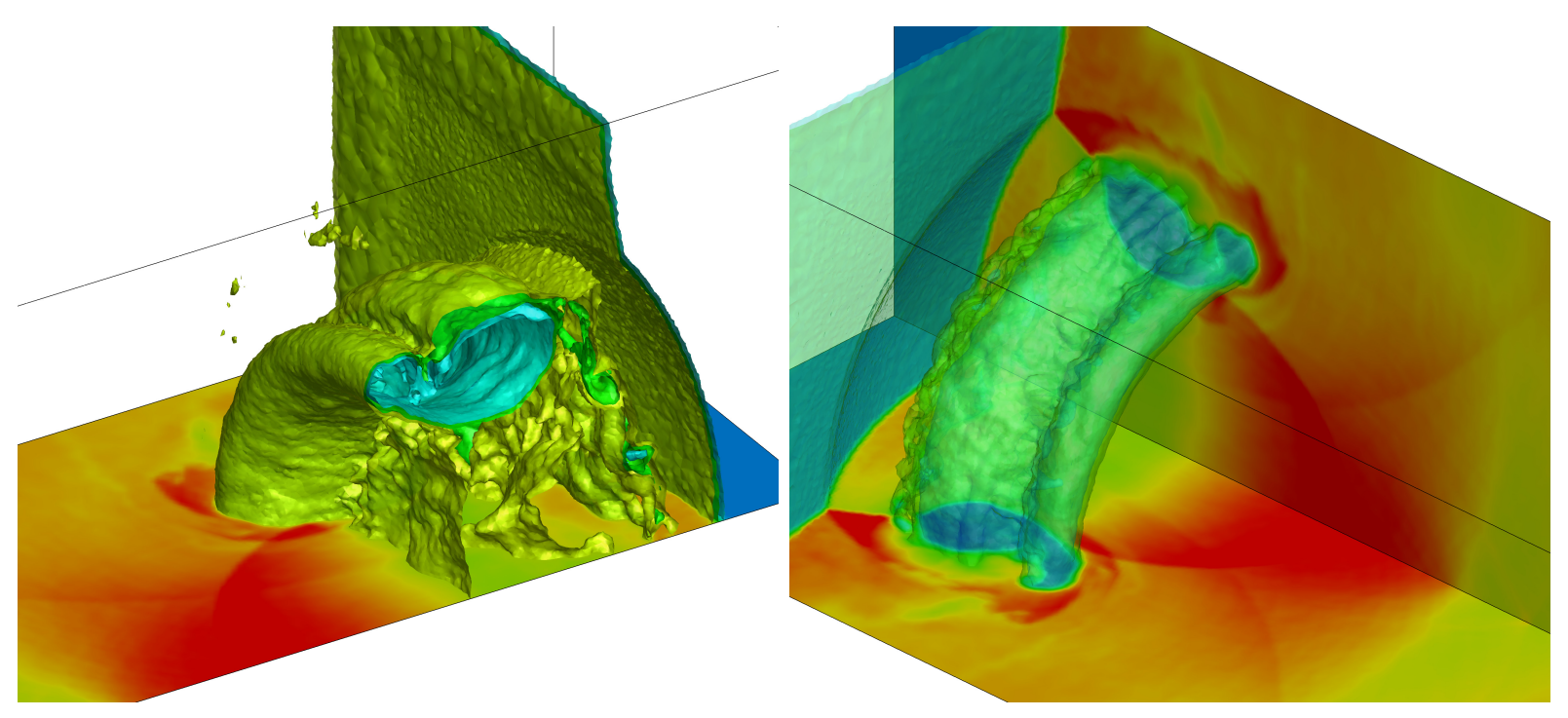

Figure 12: Close-up view around vortex torus at $t=3.5$. 


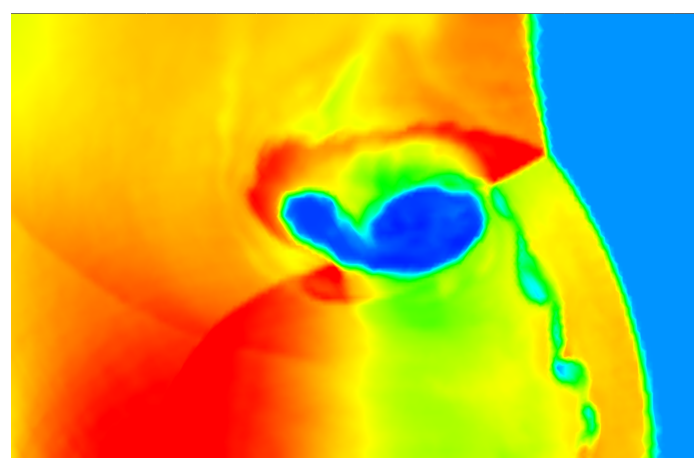

(a) DG-P2



(c) DG-P3

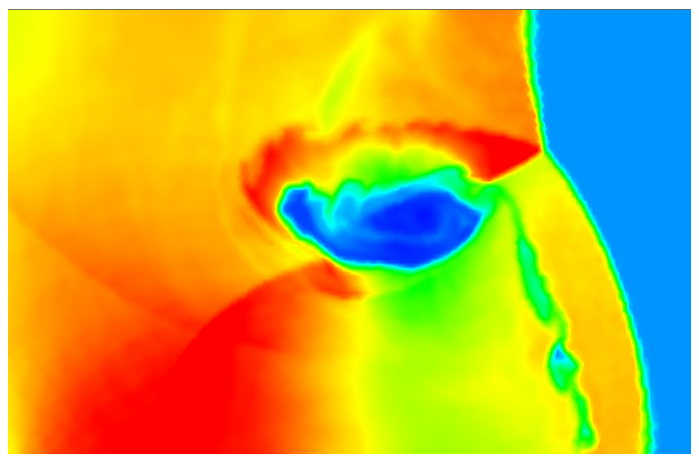

(b) CPR-P2

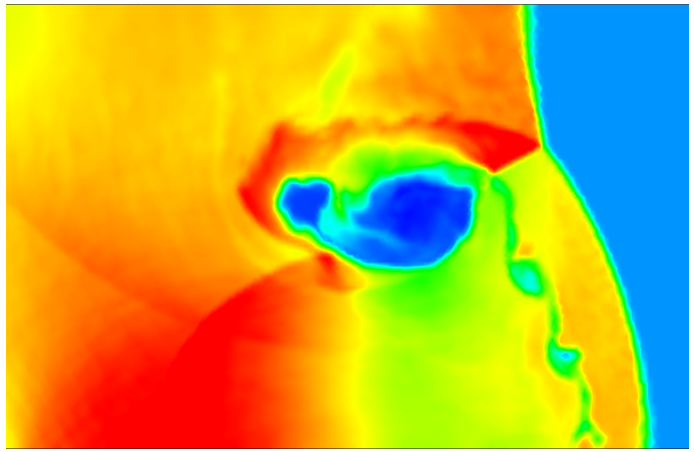

(d) CPR-P3

Figure 13: Density contours on $x-y$ surface (Shock-entropy wave interaction).

As the initial condition, a hyperbolic tangent velocity profile and a convective Mach number are imposed.

$$
\begin{gathered}
u=2.5+0.5 \tanh (2 y), \\
M_{c}=\frac{u_{1}-u_{2}}{c_{1}-c_{2}}=0.6 .
\end{gathered}
$$

An oblique shock with a shock-angle of $\beta=12^{\circ}$ is imposed on the upper boundary, and a slip wall condition is applied to the lower boundary. Periodic boundary condition is applied for both ends of z-surfaces. Fluctuation adding to the mean in-flow is given by

$$
v^{\prime}=\sum_{k=1}^{2} a_{k} \cos \left(2 \pi k t / T+z / L_{z}+\phi_{k}\right) \exp \left(-y^{2} / b\right),
$$

with a period $T=\lambda / u_{c}$, a wavelength $\lambda=30$ and a convective velocity $u_{c}=2.68$. The other parameters are as follows: $a_{1}=a_{2}=0.05, \phi_{1}=0, \phi=\pi / 2$ and $b=10 . L_{z}$, the extrusion length, is 40 . The Reynolds number and the Prandtl number are 500 and 0.72 , respectively. The computational domain is $[0,200] \times[-20,20] \times[-20,20]$. Grid system consists of 3.5 million tetrahedral elements. With Tachyon 2 supercomputer at KISTI, MPI parallel computation was performed with 512 CPUs to reach at $t=120$. For a better resolution, many filter methods have computed this problem on meshes clustered along the $y$-direction, but the present computation employs uniformly distributed triangular grids of $h=0.75$.

Figure 14 shows the density contour and iso-surfaces at $t=120$. Due to the three-dimensional perturbation (Eq. (50)), phase difference is induced along the $z$-direction. Before the oblique shock strikes the mixing layer, spanwise vortical structure is regularly developed along the $z$-direction, and after the first oblique shock-mixing layer interaction, spanwise vortical structure is noticeably deformed. After the reflected shock hits the mixing layer again, spanwise shock-vortex interaction is further developed. Figure 15 shows the density contours at $z=20$ plane. Higher-order approximation/reconstruction with MLP maintains the vortical structure along the downstream field. 




Figure 14: Density contours of three dimensional oblique shock-mixing layer interaction at $t=120$.

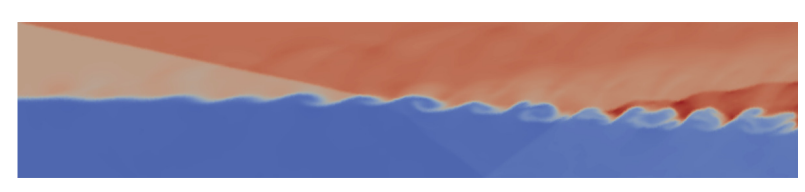

(a) DG-P2



(c) DG-P3

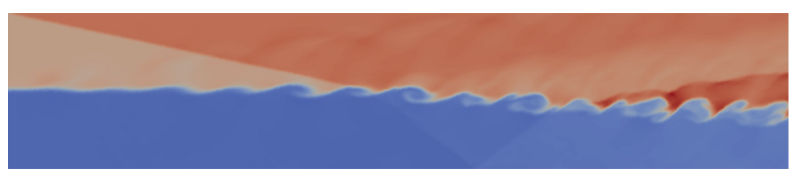

(b) CPR-P2

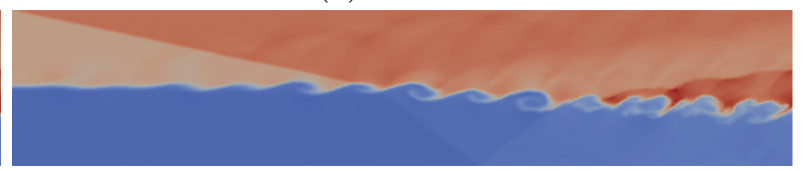

(d) CPR-P3

Figure 15: Comparison of streamwise density distributions at $z=20$. 


\section{Issues for Further Improvements}

Extensive numerical experiments validate the robustness and accuracy of the hierarchical MLP with higher-order methods in compressible inviscid and viscous flow simulations. At the same time, there are further rooms to improve and extend this limiting philosophy to deal with high-Reynolds number compressible flows around realistic configurations. Several issues are selected for discussion as follows:

\section{A. Sub-cell Resolution across Shock Waves}

As other limiter-based approaches, MLP enforces the monotonicity of cell-averaged values only. Thus, it may not completely control the sub-cell resolution around a shock wave. As observed in Figs. 4 and 5, current hierarchical MLP yields slight overshoots in the sub-cell distribution of a numerical shock. This may grow as a potential source of numerical instability if the order-of-accuracy is further increased, because the MLP condition is only imposed on the averaged value of the cell in which there are many degrees of freedom. Recently, there are some studies combining $h p$-refinement and limiters. ${ }^{45}$ This approach requires both higher-order DG/CPR and FVM solvers, and computational overheads may become serious while switching between these solvers. Unlike limiters, artificial viscosity-based approaches does not seem to suffer from this issue. Instead, there are tuning parameters to determine the diffusion, which usually relies on the flow structure, grid system and the desired order-of-accuracy. ${ }^{11}$ Despite some progresses to determine such tuning parameters, it appears that artificial viscosity is not robust enough yet to resolve very strong shock

and expansion waves. ${ }^{46,47}$ In the context of MLP concept, more reserach efforts needs to be directed to this issue by controlling the limited approximation or reconstruction.

\section{B. Convergence for Steady Flow Problems}

Up to now, the hierarchical MLP has been developed mainly for unsteady flow problems. While its performances are validated, convergence issue for steady-state problems still remains. It is well-known that slope limiters whose operations are non-differentiable, may fail to reach a convergent solution even in finite volume methods. Such non-differentiable operators are sensitive to the numerical fluctuations near shock wave and they may become stalled. While we proposed the MLP-u2 slope limiters to overcome this issue,$^{24,25}$ the troubled-cell detecting mechanism and projection operator for the DG and CPR methods are still nondifferentiable. In addition, higher-order CFD methods have a reduced damping mechanism for transient error, thus this situation may become more problematic. Implicit time integrations are preferred to compute steady flow problems, but it is quite untractable to construct an implicit operator for non-differentiable hierarchical MLP. It appears that additional smooth transition mechanism between troubled-cell and normal-cell is necessary.

\section{Interaction of Turbulent Vortices with Shock Waves}

One of the promising areas for higher-order methods is turbulent flows. Especially, researchers expect higherorder schemes to accurately resolve interactions between shock and turbulent vortices around high-speed vehicles. Recent researches attempt to calculate turbulence by Reynolds-averaged Navier-Stokes (RANS) equations with Spalart-Allmaras model ${ }^{48}$ and $k-\omega$ model, ${ }^{29}$ and by DNS/LES approaches. ${ }^{49}$ Numerical experiments reveal that current higher-order CFD methods are not robust as finite volume RANS solvers, primarily due to the nonsmoothness introduced in turbulence models. ${ }^{49}$ Recently, there are some progresses to improve the accuracy of turbulence models by modifying closure models ${ }^{48,50}$ by developing hybrid RANSLES models, ${ }^{51,52}$ or by introducing transition models. ${ }^{53}$ The enhanced resolution of higher-order methods makes it possible to accurately simulate highly unsteady turbulent flow and/or laminar-turbulent transitions with improved turbulence and/or transition models. However, there are few studies to simulate turbulent flows with shock waves with higher-order methods. Since excessive numerical viscosity of shock-capturing schemes may easily dissipate small scale vorticial structure, accurate shock-capturing schemes are indeed essential. From successful numerical experiments, we are expecting that the hierarchical MLP limiting may provide a proper dissipation-controll mechanism to capture detailed turbulent flows as well as shock waves. 


\section{Conclusions}

Guided by the MLP condition and the maximum principle, the hierarchical MLP limiting is successfully extended into the higher-order CFD methods such as the DG and CPR methods. The extended forms of the MLP condition, i.e., the augmented MLP condition and the P1-projected MLP condition, are proposed to treat the solution points near discontinuities without compromising the higher-order nature in smooth region. The uncertainty of determining a parameter for slope limiting is then eliminated by examining the behavior of local extrema near vertex point. Finally, the hierarchical MLP limiting is formulated by combining one of the extended MLP conditions with the MLP extrema detector.

Extensive computations, ranging from scalar conservation laws to multi-dimensional flow systems, are carried out up to P3 approximation to examine the capability of the hierarchical MLP methods in capturing multi-dimensional flow physics. Numerous comparisons and grid refinement tests on unstructured grids demonstrate the proposed limiting provides detailed multi-dimensional flow structures without numerical oscillations in discontinuous region, while maintaining the required accuracy in smooth region. The hierarchical MLP limiting is robust and efficient in the sense that it does not require any tuning parameter and it is applied to conservative variables without characteristic decomposition. At the same time, more efforts need to be exerted in the areas of sub-cell resolution, steady-state convergence and turbulence/transition models in order to extend the current approcch to the simulations of high-Reynolds number compressible flows around realistic configurations.

\section{Acknowledgments}

Authors appreciate the financial supports by the EDISON program through the National Research Foundation of Korea (NRF) funded by the Ministry of Science, ICT \& Future Planning (NRF-2011-0020559) and by NSL (National Space Laboratory) program through the National Research Foundation of Korea funded by the Ministry of Education, Science and Technology (NRF-2014M1A3A3A02034856). This work is also partially supported by the RoK ST\&R project of Lockheed Martin Corporation. Authors also appreciate the computing resources provided by the KISTI Supercomputing Center(KSC-2014-C3-054).

\section{References}

\footnotetext{
${ }^{1}$ Cockburn, B., Karniadakis, G. E., and Shu, C.-W., Discontinuous Galerkin Methods: Theory, Computation and Applications, Springer-Verlag Inc., 2000.

${ }^{2}$ Wang, Z. J., "High-order methods for the Euler and Navier-Stokes equations on unstructured grids," Progress in Aerospace Sciences, Vol. 43, No. 1-3, Jan. 2007, pp. 1-41.

${ }^{3}$ Vincent, P. E. and Jameson, A., "Facilitating the adoption of unstructured high-order methods amongst a wider community of fluid dynamicists," Mathematical Modelling of Natural Phenomena, Vol. 6, No. 3, May 2011, pp. 97-140.

${ }^{4}$ Wang, Z. J., Adaptive High-Order Methods in Computational Fluid Dynamics, Vol. 2, World Scientific Pub Co Inc, Jan. 2011.

${ }^{5}$ Huynh, H. T., "A flux reconstruction approach to high-order schemes including discontinuous Galerkin methods," 18th AIAA Computational Fluid Dynamics Conference, Miami, FL, 2007.

${ }^{6}$ Huynh, H. T., "A reconstruction approach to high-order schemes including discontinuous Galerkin for diffusion," $47 t h$ AIAA Aerospace Sciences Meeting Including The New Horizons Forum and Aerospace Exposition, Orlando, FL, 2009.

${ }^{7}$ Wang, Z. J. and Gao, H., "A unifying lifting collocation penalty formulation including the discontinuous Galerkin, spectral volume/difference methods for conservation laws on mixed grids," Journal of Computational Physics, Vol. 228, No. 21, Nov. 2009, pp. 8161-8186.

${ }^{8}$ Vincent, P. E., Castonguay, P., and Jameson, A., "A new class of high-order energy stable flux reconstruction schemes," Journal of Scientific Computing, Vol. 47, No. 1, June 2011, pp. 50-72.

${ }^{9}$ Castonguay, P., Vincent, P. E., and Jameson, A., "A new class of high-order energy stable flux reconstruction schemes for triangular elements," Journal of Scientific Computing, Vol. 51, No. 1, June 2011, pp. 224-256.

${ }^{10}$ Cook, A. W. and Cabot, W. H., "Hyperviscosity for shock-turbulence interactions," Journal of Computational Physics, Vol. 203, No. 2, 2005, pp. 379-385.

${ }^{11}$ Persson, P.-o. and Peraire, J., "Sub-cell shock capturing for discontinuous Galerkin methods," 44th AIAA Aerospace Sciences Meeting and Exhibit, No. January, Reno, Nevada, 2006.

${ }^{12}$ Cockburn, B. and Shu, C.-W., "The Runge-Kutta discontinuous Galerkin method for conservation laws V: multidimensional systems," Journal of Computational Physics, Vol. 141, 1998, pp. 199-224.

${ }^{13}$ Biswas, R., Devine, K. D., and Flaherty, J. E., "Parallel, adaptive finite element methods for conservation laws," Applied Numerical Mathematics, Vol. 14, No. 1-3, 1994, pp. 255-283.

${ }^{14}$ Burbeau, A., Sagaut, P., and Bruneau, C.-H., "A problem-independent limiter for high-order Runge-Kutta discontinuous Galerkin methods," Journal of Computational Physics, Vol. 169, No. 1, May 2001, pp. 111-150.
} 
${ }^{15}$ Krivodonova, L., "Limiters for high-order discontinuous Galerkin methods," Journal of Computational Physics, Vol. 226, No. 1, Sept. 2007, pp. 879-896.

${ }^{16}$ Yang, M. and Wang, Z. J., "A parameter-free generalized moment limiter for high-order methods on unstructured grids," Advances in Applied Mathematics and Mechanics, Vol. 1, No. 45, 2009, pp. 451-480.

${ }^{17} \mathrm{Xu}, \mathrm{Z}$., Liu, Y., and Shu, C.-W., "Hierarchical reconstruction for discontinuous Galerkin methods on unstructured grids with a WENO-type linear reconstruction and partial neighboring cells," Journal of Computational Physics, Vol. 228, No. 6, April 2009, pp. 2194-2212.

${ }^{18} \mathrm{Xu}, \mathrm{Z}$., Liu, Y., Du, H., Lin, G., and Shu, C.-W., "Point-wise hierarchical reconstruction for discontinuous Galerkin and finite volume methods for solving conservation laws," Journal of Computational Physics, Vol. 230, No. 17, July 2011, pp. 6843-6865.

${ }^{19}$ Zhu, J., Qiu, J., Shu, C.-W., and Dumbser, M., "Runge-Kutta discontinuous Galerkin method using WENO limiters II: Unstructured meshes," Journal of Computational Physics, Vol. 227, No. 9, April 2008, pp. 4330-4353.

${ }^{20}$ Luo, H., Baum, J. D., and Löhner, R., "A discontinuous Galerkin method based on a Taylor basis for the compressible flows on arbitrary grids," Journal of Computational Physics, Vol. 227, No. 20, Oct. 2008, pp. 8875-8893.

${ }^{21} \mathrm{Zhu}$, J. and Qiu, J., "Hermite WENO schemes and their application as limiters for Runge-Kutta discontinuous Galerkin method, III: unstructured meshes," Journal of Scientific Computing, Vol. 39, No. 2, Jan. 2009, pp. $293-321$.

${ }^{22} \mathrm{Kim}, \mathrm{K} . \mathrm{H}$. and Kim, C., "Accurate, efficient and monotonic numerical methods for multi-dimensional compressible flows: Part II: Multi-dimensional limiting process," Journal of Computational Physics, Vol. 208, No. 2, Sept. 2005, pp. 570-615.

${ }^{23}$ Yoon, S.-H., Kim, C., and Kim, K.-H., "Multi-dimensional limiting process for three-dimensional flow physics analyses," Journal of Computational Physics, Vol. 227, No. 12, June 2008, pp. 6001-6043.

${ }^{24}$ Park, J. S., Yoon, S.-H., and Kim, C., "Multi-dimensional limiting process for hyperbolic conservation laws on unstructured grids," Journal of Computational Physics, Vol. 229, No. 3, Feb. 2010, pp. 788-812.

${ }^{25}$ Park, J. S. and Kim, C., "Multi-dimensional limiting process for finite volume methods on unstructured grids," Computers Es Fluids, Vol. 65, July 2012, pp. 8-24.

${ }^{26}$ Park, J. S. and Kim, C., "Higher-order multi-dimensional limiting strategy for discontinuous Galerkin methods in compressible inviscid and viscous flows," Computers 83 Fluids, Vol. 96, 2014, pp. 377-396.

${ }^{27}$ Park, J. S. and Kim, C., "Hierarchical multi-dimensional limiting strategy for correction procedure via reconstruction," Journal of Computational Physics, submitted for publication.

${ }^{28}$ Karniadakis, G. E. and Sherwin, S. J., Spectral/hp Element Methods for CFD, Oxford University Press, USA, 1999.

${ }^{29}$ Bassi, F., Crivellini, A., Rebay, S., and Savini, M., "Discontinuous Galerkin solution of the Reynolds-averaged NavierStokes and k-w turbulence model equations," Computers 86 Fluids, Vol. 34, No. 4?5, 2005, pp. 507-540.

${ }^{30}$ Keast, P., "Moderate-degree tetrahedral quadrature formulas," Computer Methods in Applied Mechanics and Engineering, Vol. 55, 1986, pp. 339-348.

${ }^{31} \mathrm{Gao}, \mathrm{H}$. and Wang, Z. J., "A conservative correction procedure via reconstruction formulation with the Chain-Rule divergence evaluation," Journal of Computational Physics, Vol. 232, No. 1, Jan. 2013, pp. 7-13.

${ }^{32}$ Gao, H., Wang, Z. J., and Huynh, H. T., "Differential formulation of discontinuous Galerkin and related methods for the Navier-Stokes equations," Communications in Computational Physics, Vol. 13, No. 4, 2012, pp. 1013-1044.

${ }^{33}$ Spiteri, R. J. and Ruuth, S. J., "A new class of optimal high-order strong-stability-preserving time discretization methods," SIAM Journal on Numerical Analysis, Vol. 40, No. 2, 2001, pp. 469-491.

${ }^{34}$ Krivodonova, L., Xin, J., Remacle, J.-F., Chevaugeon, N., and Flaherty, J. E., "Shock detection and limiting with discontinuous Galerkin methods for hyperbolic conservation laws," Applied Numerical Mathematics, Vol. 48, No. 3-4, March 2004, pp. 323-338.

${ }^{35}$ Karypis, G. and Kumar, V., "Multilevel k-way partitioning scheme for irregular graphs,," Journal of Parallel and Distributed Computing, Vol. 48, No. 1, 1998, pp. 96-129.

${ }^{36}$ Kim, S.-s., Kim, C., Rho, O.-H., Kyu Hong, S., and Hong, S. K., "Cures for the shock instability: Development of a shock-stable Roe scheme," Journal of Computational Physics, Vol. 185, No. 2, March 2003, pp. 342-374.

${ }^{37} \mathrm{Kim}, \mathrm{K} . \mathrm{H}$., Kim, C., and Rho, O.-H., "Methods for the accurate computations of hypersonic flows I. AUSMPW+ scheme," Journal of Computational Physics, Vol. 174, No. 1, Nov. 2001, pp. 38-80.

${ }^{38}$ Geuzaine, C. and Remacle, J.-F., "Gmsh: A 3-D finite element mesh generator with built-in pre- and post-processing facilities," International Journal for Numerical Methods in Engineering, Vol. 79, No. 11, Sept. 2009, pp. 1309-1331.

${ }^{39} \mathrm{Shu}$, C.-W. and Osher, S., "Efficient implementation of essentially non-oscillatory shock-capturing schemes, II," Journal of Computational Physics, Vol. 83, No. 1, 1989, pp. 32-78.

${ }^{40}$ Woodward, P. R. and Colella, P., "The numerical simulation of two-dimensional fluid flow with strong shocks," Journal of Computational Physics, Vol. 54, No. 1, 1984, pp. 115-173.

${ }^{41}$ Dyke, M. V., An Album of Fluid Motion, The Parabolic Press, 1982.

${ }^{42}$ Chang, S.-M. and Chang, K.-S., "On the shock-vortex interaction in Schardin's problem," Shock Waves, Vol. 10, No. 5, Nov. 2000, pp. 333-343.

${ }^{43}$ Bagabir, A. and Drikakis, D., "Mach number effects on shock-bubble interaction," Shock Waves, Vol. 11, 2000, pp. 209218.

${ }^{44}$ Yee, H. C., Sandham, N. D., and Djomehri, M. J., "Low-dissipative high-order shock-capturing methods using characteristic-based filters," Journal of Computational Physics, Vol. 150, No. 1, 1999, pp. 199-238.

${ }^{45}$ Dumbser, M., Zanotti, O., Loubere, R., and Diot, S., "A posteriori subcell limiting of the discontinuous Galerkin finite element method for hyperbolic conservation laws," Journal of Computational Physics, Vol. 278, 2014, pp. 47-75.

${ }^{46} \mathrm{Yu}, \mathrm{M}$. and Wang, Z. J., "Shock capturing for the Correction Procedure via Reconstruction method using artificial viscosity and diffusivity," ICCFD8 Paper, 2014-0079, 2014. 
${ }^{47}$ Park, J. S., Yu, M., Kim, C., and Wang, Z. J., "Comparative Study of Shock-Capturing Methods for High-Order CPR: MLP and Artificial Viscosity," ICCFD8 Paper, 2014-0067, 2014.

${ }^{48}$ Nguyen, N. C., Persson, P.-O., and Peraire, J., "RANS solutions using high order discontinuous Galerkin methods," 45th AIAA Aerospace Sciences Meeting and Exhibit, Reno, NV, 2007.

${ }^{49}$ Wang, Z., Fidkowski, K., Abgrall, R., Bassi, F., Caraeni, D., Cary, A., Deconinck, H., Hartmann, R., Hillewaert, K., Huynh, H., et al., "High-order CFD methods: current status and perspective," International Journal for Numerical Methods in Fluids, Vol. 72, No. 8, 2013, pp. 811-845.

${ }^{50}$ Allmaras, S. R., Johnson, F. T., and Spalart, P. R., "Modifications and Clarifications for the Implementation of the Spalart-Allmaras Turbulence Model," ICCFD7, No. July, 2012, pp. 9-13.

${ }^{51}$ Spalart, P., Jou, W., Strelets, M., and Allmaras, S., "Comments on the feasibility of LES for wings, and on a hybrid RANS/LES approach," Advances in DNS/LES, Vol. 1, 1997, pp. 4-8.

${ }^{52}$ Spalart, P. R., Deck, S., Shur, M., Squires, K., Strelets, M. K., and Travin, A., "A new version of detached-eddy simulation, resistant to ambiguous grid densities," Theoretical and computational fluid dynamics, Vol. 20, No. 3, 2006, pp. $181-195$.

${ }^{53}$ Langtry, R. B. and Menter, F. R., "Correlation-based transition modeling for unstructured parallelized computational fluid dynamics codes," AIAA journal, Vol. 47, No. 12, 2009, pp. 2894-2906. 